\title{
Dynamic crack initiation assessment with the coupled criterion
}

Aurélien Doitrand ${ }^{\mathrm{a}, *}$, Gergely Molnár ${ }^{\mathrm{b}}$, Dominique Leguillon $^{\mathrm{c}}$, Eric Martin $^{\mathrm{d}}$, Nicolas Carrère

${ }^{a}$ Univ Lyon, INSA-Lyon, UCBL, CNRS UMR5510, MATEIS, F-69621, France

${ }^{b}$ Univ Lyon, CNRS, INSA-Lyon, UCBL, UMR5259, LaMCoS, F-69621, France

${ }^{c}$ Institut Jean le Rond d'Alembert, Sorbonne Université, CNRS UMR 7190, F-75005, France

${ }^{d}$ Laboratoire des Composites Thermo-Structuraux, CNRS UMR 5801, Université de Bordeaux, F-33600, France

${ }^{e}$ IRDL, CNRS UMR6027, Brest, France

\begin{abstract}
An extension of the coupled criterion (CC) of finite fracture mechanics is established in order to assess dynamic crack initiation. The main change compared to the classical quasi-static approach consists in considering the crack velocity profile during initiation instead of assuming an instantaneous crack initiation. The proposed approach enables the study of dynamic crack initiation under either quasi-static or dynamic loading. It is illustrated on several examples including transverse cracking in laminates, crack initiation in drilled specimen under quasi-static tension or compression and on a V-notch specimen subjected to time-dependent loading. The dynamic CC predicts crack initiation over a finite length occurring in a given time depending on the crack velocity. The influence of the initiation crack velocity profile is significative provided crack velocity is large enough. Taking into account dynamic effects enables a better representation of experimentally observed variation of initiation stress as a function of hole size under tensile loading in drilled hole specimens.
\end{abstract}

Keywords: Coupled criterion; crack initiation; dynamic

\section{Introduction}

Fracture in brittle materials can be addressed using Linear Elastic Fracture Mechanics (LEFM) $[1,2]$. This theory is based on the major assumption of the existence of a crack and provides a framework for studying its propagation. However, this assumption limits the study to crack propagation and fails to predict crack initiation.

Considering finite rather than infinitesimal crack increments, Finite Fracture Mechanics (FFM)

\footnotetext{
*Corresponding author

Email address: aurelien.doitrand@insa-lyon.fr (Aurélien Doitrand)
} 
$[3,4]$ provided a breakthrough in crack initiation study. In this framework, Leguillon [5] set up the coupled criterion (CC) for crack initiation prediction. The basic principle of the $\mathrm{CC}$ is the simultaneous fulfillment of two separate conditions to predict crack initiation. It states that, on one hand, before initiation, the stress must be larger than the material strength all over the initiation crack path. On the other hand, the potential energy release must be larger than the crack surface creation energy. It results in two inequalities that both provide, for a given loading level, a range of crack lengths for which the corresponding criteria are fulfilled. The initiation loading level is thus determined as the minimum loading for which both inequalities are simultaneously met, which also provides the initiation crack length.

Since its introduction, the $\mathrm{CC}$ has proved to be a robust and efficient approach to predict crack initiation in a large range of materials and configurations, summarized in the review paper by Weissgraeber et al. [6]. Originally developed in a linear elastic framework under small deformation assumption and for 2D quasi-static cases, more recent works about the $\mathrm{CC}$ concern:

- Accounting for nonlinearities such as in the case of damaged materials $[7,8]$ or nonlinear elastic behavior under small $[9,10,11]$ and large deformation [12],

- Numerical implementations in Finite Element (FE) codes [8, 13, 14, 15],

- Comparisons with other fracture models such as, e.g. cohesive zone models $[16,17,18,19,20]$ or phase field approach for fracture [21, 22, 23].

- Post-processing of experimental tests at micro- $[24,25,26]$ or nano- [27] scale,

- Its extension and application to 3D cases [28, 29, 30, 31, 32].

Experimental observations of crack initiation in PMMA rhombus hole specimens under quasistatic loading highlighted abrupt crack initiation [33], which means that a crack nucleates and propagates in an unstable manner over a finite size in short time and displacement increments. The initiation time and crack lengths were however not quantified for several reasons. First, the frame acquisition frequency was not sufficiently high and in addition, crack nucleation was followed by unstable propagation up to an arrest length. It was therefore not straightforward to distinguish crack initiation and unstable propagation phases in the experiments.

Theoretical studies providing a framework for dynamic fracture mechanics were proposed [34, 35, 36]. Dynamic failure may also be assessed using previously mentioned numerical approaches such 
as phase field $[37,38,39]$ or cohesive zone models [20, 40]. To the authors' knowledge, accounting for crack initiation dynamics in the $\mathrm{CC}$, which was identified as a possible extension of the $\mathrm{CC}$ in the review paper by Weissgraeber [6], has not yet been addressed. Indeed, crack initiation description using the $\mathrm{CC}$ is based on the assumption of instantaneous crack initiation. This is actually an approximation of the abrupt dynamic crack nucleation occurring in a short time increment. Recently, Laschuetza and Seelig [41] investigated dynamic cohesive fracture of a plate with hole under static pre-stress compared to quasi-static FFM results, noticing that FFM generally predicts an excess of energy after crack initiation $\left(G>G_{c}\right.$, where $G$ is the quasi-static energy release rate (ERR) and $G_{c}$ the material toughness). By assuming that this energy excess corresponds to the

neglected inertial effects so that the dynamic $\operatorname{ERR}\left(G_{d y n} \approx\left(1-\frac{\dot{\ell}}{c_{R}}\right) G\right.$, where $\dot{\ell}$ is the crack velocity and $c_{R}$ the Rayleigh wave speed) is actually equal to $G_{c}$, they estimated the velocity of the crack tip during the finite crack increment.

The objective of this work is to take into account crack initiation under dynamic conditions in the $\mathrm{CC}$. The quasi-static approach of the $\mathrm{CC}$ is recalled and extended to take into account dynamic crack initiation in Section 2. The example of crack propagation in infinite media is addressed in section 3. The $\mathrm{CC}$ is then applied to assess dynamic crack initiation under quasi-static loading in laminates (Section 4), in drilled hole specimens (Sections 5 and 6), including the influence of the crack velocity profile (Section 6), as well as in the case of a time-dependent loading (Section 7).

\section{The coupled criterion}

In the sequel, we will consider 2D plane strain assumption but the reasoning can also be extended to the 3D case. We consider linear elastic material behavior under small deformation, even if the reasoning can also be extended to account for material or geometric nonlinearities. We consider a single crack following a prescribed path. The CC overcomes the limitation of LEFM regarding crack initiation assessment since it does not require any pre-existing crack but is able to assess the nucleation of a crack. It is based on the simultaneous fulfillment of stress and energy requirements. The first condition of the $\mathrm{CC}$ ensures that the stress is larger than the material tensile strength all over the crack path before crack initiation, which writes:

$$
\sigma(\ell, U) \geqslant \sigma_{c} \forall 0 \leqslant \ell \leqslant \ell_{c}
$$


where $U$ represents the prescribed loading or displacement and $\ell_{c}$ is the a priori unknown initiation length that is determined through the combination of stress and energy conditions. The second condition is obtained by balancing the changes in the external force work $\left(W_{\text {ext }}\right)$, the elastic strain $\left(W_{p}=\frac{1}{2} \int_{V} \underline{\sigma}: \underline{\varepsilon} d V\right)$, kinetic $\left(W_{k}\right)$, and crack surface creation $\left(G_{c} \ell\right.$, where $G_{c}$ is the material fracture toughness and $\ell$ the crack length) energy between the states prior to and after crack initiation.

$$
\Delta W_{e x t}(\ell, U)-\Delta W_{p}(\ell, U)-\Delta W_{k}(\ell, U)=G_{c} \ell
$$

In 2D, the left side of Eq. (2) must be understood per unit thickness of the specimen. Note that when the stress $\sigma$ is depicted as a function of $\ell, \ell$ represents the position along the prescribed crack path before initiation whereas it corresponds to the crack length in the energy condition. The two conditions given in Eqs. (1) and (2) have to be simultaneously fulfilled so that crack initiation can occur. It reverts to determining the minimum prescribed loading or displacement and the corresponding initiation crack length satisfying both equations.

\subsection{Quasi-static approach}

Under quasi-static loading, the kinetic energy before crack initiation is zero thus crack initiation leads to an increase in kinetic energy $\Delta W_{k} \geqslant 0$, so that the energy condition given in Eq. (2) yields the following inequality:

$$
G_{i n c}(\ell, U)=\frac{\Delta W_{e x t}(\ell, U)-\Delta W_{p}(\ell, U)}{\ell} \geqslant G_{c}
$$

where $G_{i n c}$ is called the incremental energy release rate (IERR). Note that, if the boundary conditions exclusively consist of prescribed displacements, $\Delta W_{\text {ext }}=0$ and $G_{\text {inc }}=-\Delta W_{p} / \ell$. Under linear elasticity and small deformation assumptions, the stress is proportional to the prescribed loading and the IERR is proportional to the square of prescribed loading:

$$
\left\{\begin{array}{l}
G_{i n c}(\ell, U)=A(\ell) U^{2} \geqslant G_{c} \\
\sigma(\ell, U)=k(\ell) U \geqslant \sigma_{c}
\end{array}\right.
$$


From these two equations the initiation loading and crack length can be determined as :

$$
\left\{\begin{array}{c}
U_{c}=\min _{\ell}\left\{\max \left(\sqrt{\frac{G_{c}}{A(\ell)}}, \frac{\sigma_{c}}{k(\ell)}\right)\right\} \\
\ell_{c}=\underset{\ell}{\operatorname{argmin}}\left\{\max \left(\sqrt{\frac{G_{c}}{A(\ell)}}, \frac{\sigma_{c}}{k(\ell)}\right)\right\}
\end{array}\right.
$$

Under quasi-static loading conditions, the CC predicts that the crack instantaneously jumps over a finite increment at initiation. Depending on the ERR variation, subsequent unstable crack propagation may occur and can be assessed using classical LEFM, leading to a possible crack arrest length. Experimentally, it is not straightforward to distinguish crack initiation from the subsequent unstable crack propagation since they both occur in a short time increment. Other approaches such as cohesive zone modeling or phase field for fracture also lead to the conclusion that a crack jump to an arrest length occurs at initiation [20, 23]. Nevertheless, these methods do not distinguish initiation and unstable crack propagation phases but they enable estimating crack length variation as a function of time during the initiation/unstable propagation phase.

From an implementation point of view under linear elasticity and small deformation assumption, the CC requires several calculations with different crack lengths (obtained by releasing Dirichlet boundary conditions on the nodes along the crack path). It allows estimating functions $k(\ell)$ and $A(\ell)$, exploiting the stress and potential energy proportionality respectively to the prescribed loading and to the square of prescribed loading. Once computed, crack initiation length and loading level are obtained using Eq. (5) for any $\left(G_{c}, \sigma_{c}\right)$ couples. More details about the FE implementation of the quasi-static approach of the CC are given in [14].

\subsection{Dynamic approach under quasi-static loading}

Under quasi-static conditions, the stress fields do not depend on loading rate but only on the loading magnitude. It means that even if dynamic crack propagation under quasi-static loading is considered, the stress condition remains unchanged compared to the quasi-static approach. It ensures that the stress is larger than the material strength all over the pre-supposed crack path before initiation. The main difference between quasi-static and dynamic approaches of the CC under quasi-static loading concerns the description of the crack length variation as a function of time. In the quasi-static approach, we assume that crack initiation occurs instantaneously. In practice, it occurs in a short time increment during which the crack length jumps from 0 to $\ell_{c}$ (or from $l_{0}$ to $\ell_{c}$ in the particular case of a pre-existing crack). The dynamic approach of the CC 


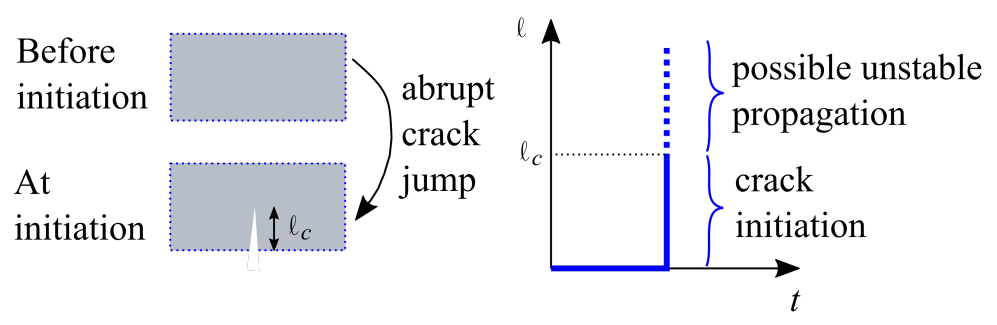

(a) (b)

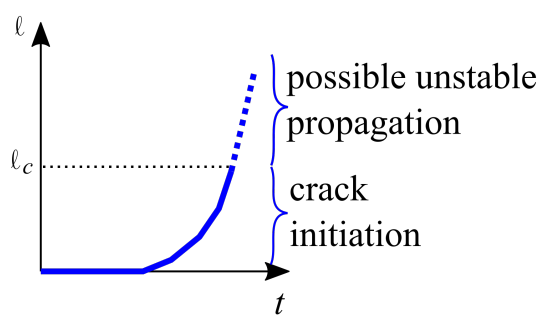

(c)

Figure 1: (a) Abrupt crack initiation and corresponding crack length variation as a function of time in (b) the quasistatic (instantaneous initiation) or (c) the dynamic approach (initiation following a velocity profile $v_{\text {crack }}(t)=\frac{d \ell(t)}{d t}$ of the CC.

describes the progressive extension to the initiation length, which is considered to happen following a certain velocity profile $v_{\text {crack }}(t)$ corresponding to the time derivative of the crack length $\ell(t)$ (Fig. 1).

It requires a new definition of the IERR so that the energy condition takes into account the kinetic energy variation due to crack initiation:

$$
G_{\text {inc }}(\ell(t), U)=\frac{\Delta W_{\text {ext }}(\ell(t), U)-\Delta W_{p}(\ell(t), U)-\Delta W_{k}(\ell(t), U)}{\ell(t)}=G_{c}\left(v_{\text {crack }}(t)\right)
$$

This definition is actually consistent with the quasi-static IERR definition (Eq. (3)) since in the quasi-static approach the kinetic energy variation is neglected. Other energy dissipation sources (such as, e.g., material plasticity or diffuse damage) can also be taken into account into the energy balance (Eq. (2)) and thus in the definition of the IERR. The energy criterion now writes as an equality rather than an inequality in the quasi-static approach. Also note that in Eq. (6), the fracture toughness may depend on the crack velocity. Indeed, a sufficiently large crack velocity may result in the creation of local microcrack branches emerging from the main crack [42, 43, 44, 45], thus increasing the total created crack surface. Branching of the main crack may also be observed if the crack velocity reaches a critical value. Therefore, the larger the crack velocity, the larger the apparent material fracture toughness.

The main implementation difference compared to the quasi-static approach concerns the crack length variation as a function of time. It requires a dynamic solution and thus two extra inputs, namely the material density $\rho$ and the crack velocity profile $v_{\text {crack }}(t)=d l(t) / d t$ to define the progressive node unbuttoning as a function of time. During each node unbuttoning step, the Dirichlet condition release induces a reaction force decrease to zero for the corresponding degree 
of freedom. A linear force decrease as a function of time is used. Similarly to the potential energy, the kinetic energy is proportional to the square prescribed loading under linear elasticity and small deformation assumption. It is basically computed as a function of the velocity $(\dot{u})$ field as:

$$
W_{k}=\frac{1}{2} \int_{V} \rho(\overrightarrow{\dot{u}} \cdot \overrightarrow{\dot{u}}) d V
$$

It yields that the IERR is also proportional to the square prescribed loading. Therefore, similarly to the quasi-static approach, several calculations with different crack lengths at a given loading are sufficient to determine the initiation crack length and loading level using Eq. (5).

\subsection{Transient dynamic loading}

Material density and crack velocity profile are also required as inputs similarly to the dynamic approach under quasi-static loading. Under dynamic loading conditions (such as a stress wave or rapid loading), the prescribed loading $U$ depends on time as well as the stress fields so that the stress conditions writes:

$$
\sigma(\ell, U(t)) \geqslant \sigma_{c}, \forall 0 \leqslant \ell \leqslant \ell_{c}
$$

The energy condition is also modified accounting for the time dependency of the loading:

$$
G_{i n c}(\ell(t), U(t))=\frac{\Delta W_{e x t}(\ell(t), U(t))-\Delta W_{p}(\ell(t), U(t))-\Delta W_{k}(\ell(t), U(t))}{\ell(t)}=G_{c}\left(v_{\text {crack }}(t)\right)
$$

Therefore, the property of stress and energy proportionality to respectively the prescribed loading and the square of prescribed loading is no longer valid. Similarly to CC application involving nonlinear material behaviors $[10,11,12,13]$, the stress and the energy conditions must therefore be evaluated for several loading levels in order to determine the minimum time $t_{c}$ corresponding to a loading level $U_{c}=U\left(t_{c}\right)$ for which both conditions are simultaneously fulfilled. It reverts to solving the following problem:

$$
U_{\mathrm{c}}=U\left(t_{c}\right) \text { where } t_{c}=\min \left\{t, \exists \ell,\left(\frac{G_{i n c}(\ell(t), U(t))}{G_{c}} \geq 1\right) \wedge\left(\frac{\sigma(\ell(t), U(t))}{\sigma_{c}} \geq 1\right)\right\}
$$

The initiation crack length $\ell_{c}=\ell\left(t_{c}\right)$ thus verifies :

$$
\min \left(\frac{G_{i n c}\left(\ell_{c}, U_{\mathrm{c}}\right)}{G_{c}}, \frac{\sigma\left(\ell_{c}, U_{\mathrm{c}}\right)}{\sigma_{c}}\right)=1
$$


In terms of implementation, additional calculations compared to the case of quasi-static loading conditions are thus required to solve the CC. The following procedure can be followed in order to determine the initiation crack length and loading level:

- For each time step, determine the set of admissible crack lengths for which the stress criterion is reached.

- For a given time step, if the set of admissible crack lengths is not empty, compute the energy variations as a function of these crack lengths given the crack velocity profile.

- Evaluate if the energy criterion is fulfilled for any of the admissible crack length.

- If not, repeat the procedure for the next time step until both criteria are fulfilled for a given crack length, corresponding to the initiation crack length.

\section{Numerical considerations - crack propagation in infinite media under remote stress}

The dynamic approach of the $\mathrm{CC}$ requires the computation of the stress prior to crack initiation and of the IERR as a function of the crack length. Whereas the former is straightforward, the latter requires the description of crack propagation following a given velocity profile. In this section, we assess the ability to compute the energy condition of the coupled criterion.

\subsection{Crack growth at a constant velocity}

We first study the example of mode I crack propagation at a constant velocity under remote stress in infinite medium. The material properties are $E=8.4 \mathrm{GPa}, \nu=0.24$ and $\rho=1300 \mathrm{~kg} / \mathrm{m}^{3}$. Under dynamic conditions, the $\operatorname{ERR}\left(G^{d y n}\right)$ and the stress intensity factor $\left(K_{I}^{d y n}\right)$ depend not only

on the crack length $\ell$ but also on its velocity $v_{\text {crack }}=d \ell / d t$. They are related to each other through the following expression [34]:

$$
G^{d y n}\left(\ell, v_{\text {crack }}\right)=\frac{1-\nu^{2}}{E} A_{I}\left(v_{\text {crack }}\right) K_{I}^{d y n}\left(\ell, v_{\text {crack }}\right)^{2}
$$

where:

$$
\left\{\begin{array}{l}
A_{I}\left(v_{\text {crack }}\right)=\frac{v_{\text {crack }}^{2} \alpha_{d}}{(1-\nu) c_{s}^{2} D\left(v_{\text {crack }}\right)}, \\
D\left(v_{\text {crack }}\right)=4 \alpha_{s} \alpha_{d}-\left(1+\alpha_{s}^{2}\right)^{2}, \\
\alpha_{d}=\sqrt{1-\frac{v_{c r a c k}^{2}}{c_{d}^{2}}} \\
\alpha_{s}=\sqrt{1-\frac{v_{\text {crack }}^{2}}{c_{s}^{2}}}
\end{array}\right.
$$


In Eq. (13), $c_{s}$ and $c_{d}$ respectively are the shear and and longitudinal wave velocities. Moreover, the dynamic ERR and stress intensity factors are related to their quasi-static counterpart ( $G$ and $\left.K_{I}\right)$ as

$$
\left\{\begin{array}{l}
G^{d y n}\left(\ell, v_{\text {crack }}\right)=g\left(v_{\text {crack }}\right) G(\ell) \\
K_{I}^{d y n}\left(\ell, v_{\text {crack }}\right)=\xi\left(v_{\text {crack }}\right) K_{I}(\ell) \\
G(\ell)=\frac{1-\nu^{2}}{E} K_{I}(\ell)^{2}
\end{array}\right.
$$

Thus the functions $g$ and $\xi$ are related by $g\left(v_{\text {crack }}\right)=A_{I}\left(v_{\text {crack }}\right) \xi\left(v_{\text {crack }}\right)^{2}$. They can be approximated by $g\left(v_{\text {crack }}\right) \approx 1-\frac{v_{c r a c k}}{c_{R}}$ and $\xi\left(v_{\text {crack }}\right) \approx\left(1-\frac{v_{\text {crack }}}{c_{R}}\right) /\left(\sqrt{1-\frac{v_{\text {crack }}}{c_{d}}}\right)$ where $c_{R}$ represents the Rayleigh wave speed. Therefore, $A_{I}$ can also be approximated by $A_{I} \approx \frac{g}{\xi^{2}} \approx\left(1-\frac{v_{\text {crack }}}{c_{d}}\right) /\left(1-\frac{v_{\text {crack }}}{c_{R}}\right)$. Note that the previous expressions are given for the ERR $G$ whereas the energy condition of the CC involves the IERR $\left(G_{i n c}\right)$ rather than $G$. However, both quantities are related through the expression:

$$
G=G_{i n c}+\ell \frac{d G_{i n c}}{d \ell}
$$

which therefore enables the calculation of $G_{i n c}$ from $G$ and vice-versa.

In the studied example of a crack propagating at constant velocity, the quasi-static ERR can be computed analytically as:

$$
G(l)=\frac{\left(1-\nu^{2}\right) \sigma_{0} \pi \ell}{E}
$$

The dynamic ERR can be approximated by $G^{d y n} \approx\left(1-\frac{v_{\text {crack }}}{c_{R}}\right) G$ [34]. It is necessary to ensure that FE calculations provide a good estimate of the ERR (and thus of its incremental counterpart) for a given crack velocity in order to correctly compute the energy condition of the coupled criterion. In FE simulations, crack advance is obtained by a progressive crack unbuttoning following a prescribed velocity profile in order to compute the elastic strain $\left(W_{p}\right)$ and kinetic $\left(W_{k}\right)$ energies. Then, the dynamic ERR and the IERR are calculated as:

$$
\left\{\begin{array}{l}
G=\frac{d W_{e x t}}{d l}-\frac{d W_{p}}{d l}-\frac{d W_{k}}{d l} \\
G_{i n c}=\frac{\Delta W_{e x t}}{\Delta l}-\frac{\Delta W_{p}}{\Delta l}-\frac{\Delta W_{k}}{\Delta l}
\end{array}\right.
$$

\subsection{Influence of time increments and mesh size}

The ERR is computed by successively unbuttoning nodes along the crack path. Uniform mesh consisting of linear four-noded elements are used, 20 elements are set along the crack propagation zone. Each node release is performed in a given time depending on the mesh size in order to ensure 


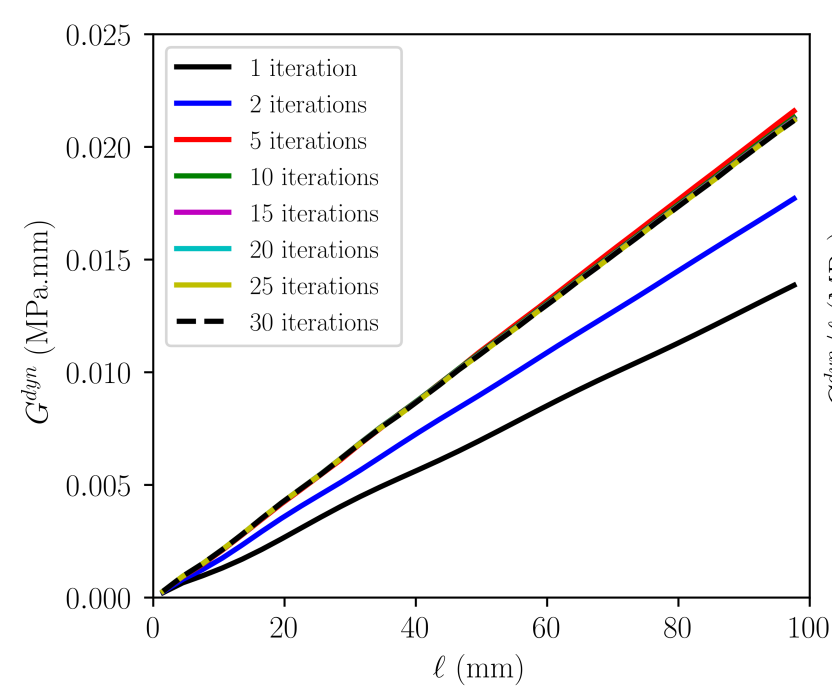

(a)

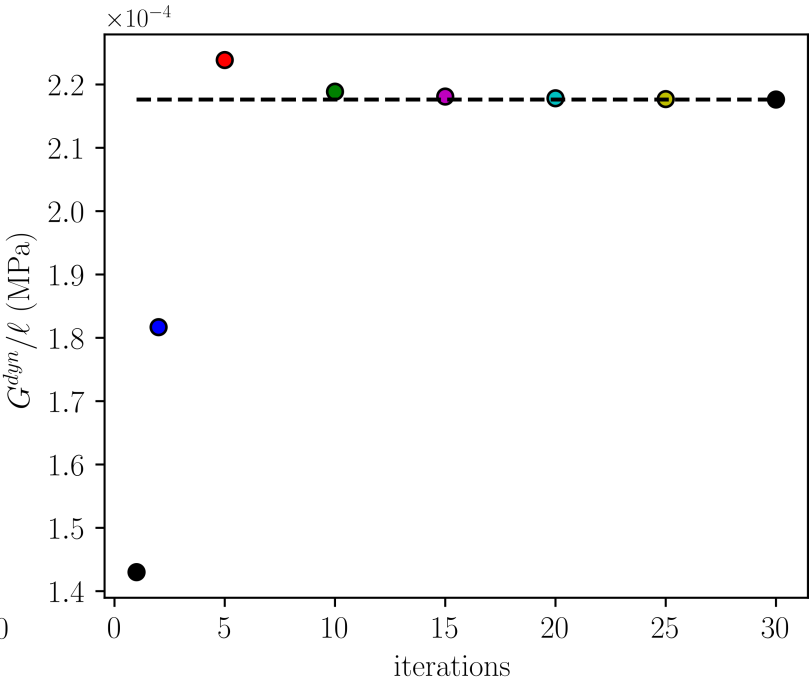

(b)

Figure 2: (a) Energy release rate as a function of crack length and (b) Dynamic energy release rate to crack length ratio obtained for $v_{\text {crack }}=0.5 c_{R}$ crack velocity and several number of time iterations during one node release. The dashed line indicates the value obtained for 30 iterations.

the prescribe crack velocity profile. We first study the influence of the number of time increments during one node release step for a given constant crack velocity $v_{\text {crack }}=0.5 c_{R}$. Fig. 2a shows the ERR as a function of the crack length obtained for several number of iterations during the release of one node. A too small number of iterations leads to underestimating (or overestimating) the ERR. The slope of the ERR variation as a function of the crack length converges when increasing the number of iterations during one node release (Fig. 2b). For 20 iterations or more per node release, ERR difference smaller than $0.1 \%$ are obtained. In the following, we thus set 20 iterations per node unbuttoning. We also investigate the influence of mesh size on the ERR calculation. Fig. 3 shows the ERR variation as a function of crack length for several mesh sizes for $v_{\text {crack }}=0.4 c_{R}$ constant crack velocity. The different mesh sizes corresponds to a number of elements within the crack propagation area comprised between 11 and 33. The corresponding ERR as a function of the crack length are shown in Fig. 3. The different mesh sizes lead to ERR differences smaller than $2 \%$. Nevertheless, the CC implementation requires a sufficiently fine mesh in order to catch the initiation length that results from coupling both energy and stress conditions. It is known from the $\mathrm{CC}$ quasi-static solution that the initiation length is a fraction of the material characteristic length $l_{m a t}=\frac{E G_{c}}{\left(1-\nu^{2}\right) \sigma_{c}^{2}}$. It is thus recommended that the minimum mesh size along the crack path is smaller than $l_{\text {mat }} / 40$ [14]. In the CC dynamic approach, it is expected that the larger the crack velocity, the smaller the potential energy difference and the larger the kinetic energy variation. Therefore, the larger the crack velocity, the smaller the IERR and thus the larger the initiation 


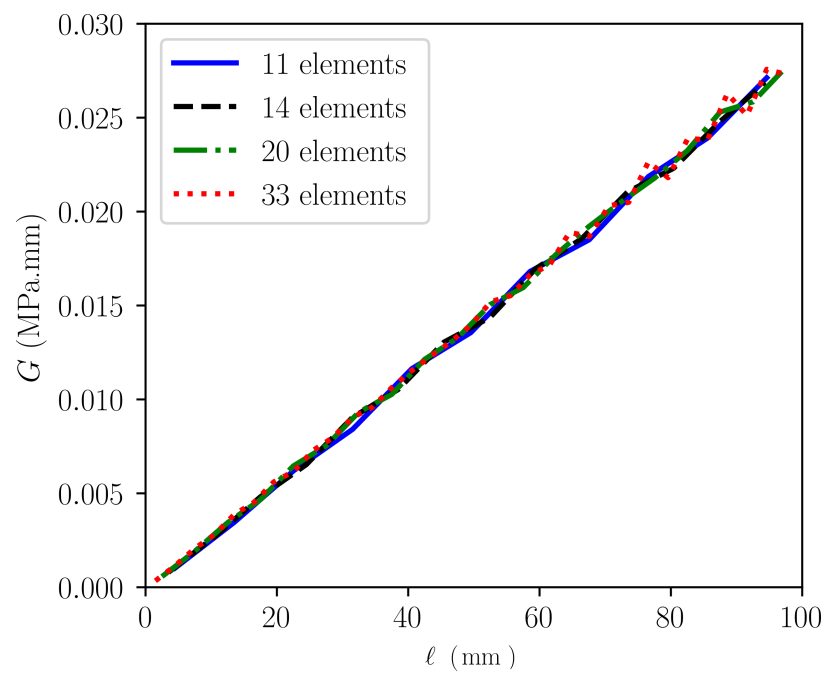

Figure 3: (a) Energy release rate as a function of crack length obtained for $v_{\text {crack }}=0.4 c_{R}$ crack velocity and several mesh sizes.

crack length. We thus conclude that the same mesh size recommendations as in the CC quasi-static approach [14] can be followed in the CC dynamic approach.

\subsection{Influence of crack velocity}

The influence of crack velocity (assuming a constant crack velocity profile) is now considered. We compute the dynamic ERR (Eq. 17) as a function of the crack length for several crack velocities (Fig. 4a). For small crack velocity to Rayleigh velocity ratios, we observe some small oscillations in the ERR. Nevertheless, the obtained ERR variations as a function of crack length can be considered as linear, the ratio of their slope to the slope of the quasi-static ERR is shown in Fig. 4b.

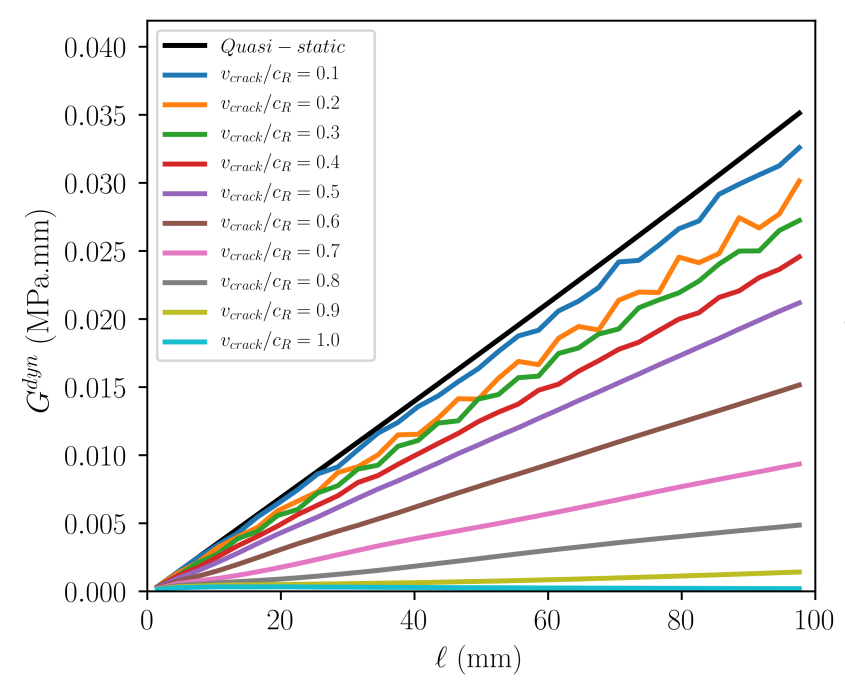

(a)

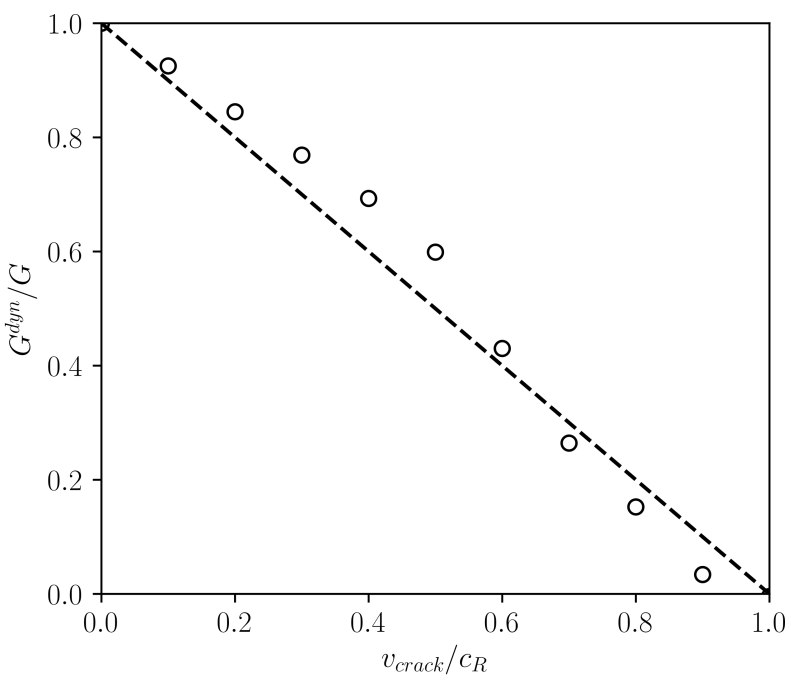

(b)

Figure 4: (a) Energy release rate as a function of crack length for several crack velocities. (b) Dynamic $\left(G^{d y n}\right)$ to quasi-static $(G)$ energy release rate ratio as a function of crack velocity to Rayleigh velocity ratio. 
Theoretically, a linear decreasing variation of the dynamic to quasi-static ERR ratio as a function of the crack velocity to Rayleigh velocity ratio is expected [34, 46]. The variation obtained from FE calculations is close to the expected theoretical variation, with a slight overestimate for crack velocities between $0.2 c_{R}$ and $0.6 c_{R}$.

Stiffness proportional damping may be used in order to reduce numerical high-frequency noise without having any significant effect on the lower frequency response [47]. This numerical damping is expected to reduce the oscillations due to the node release [48]. Kopp et al. [49] recommend to use $\beta \times v_{\text {crack }}=0.1 \mathrm{~mm}$, where $\beta$ is the damping coefficient. The influence of this parameter is shown in Fig. 5 for a $v_{\text {crack }}=0.1 c_{R}$ constant crack velocity. Introducing damping allows reducing the small oscillations in the ERR variation as a function of the crack length. However, a too large value of the damping coefficient results in a decrease in the ERR whereas it almost remains constant for sufficiently small values. Fig. 6 shows the ERR variation as a function of the crack length as well as the dynamic to quasi-static ERR ratio for several crack velocities obtained for $\beta v_{\text {crack }}=0.1 \mathrm{~mm}$, which is close to that obtained without damping (Fig. 4) while reducing the amplitude of the oscillations.

In this first example we computed the dynamic ERR using Eq. (17) and validated its calculation by comparison to an analytical solution [34]. As explained previously, $G_{i n c}$ can be either derived from $G$ using Eq. (15) or also directly computed from the finite element solution. Another possibility to compute $G^{d y n}$ is the use of a dynamic J-integral $[50,51]$ which writes:

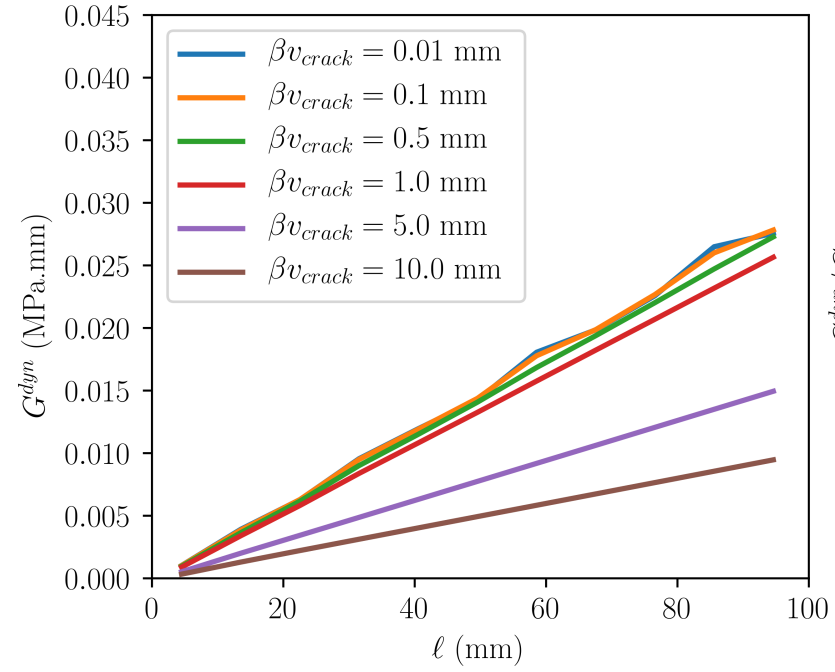

(a)

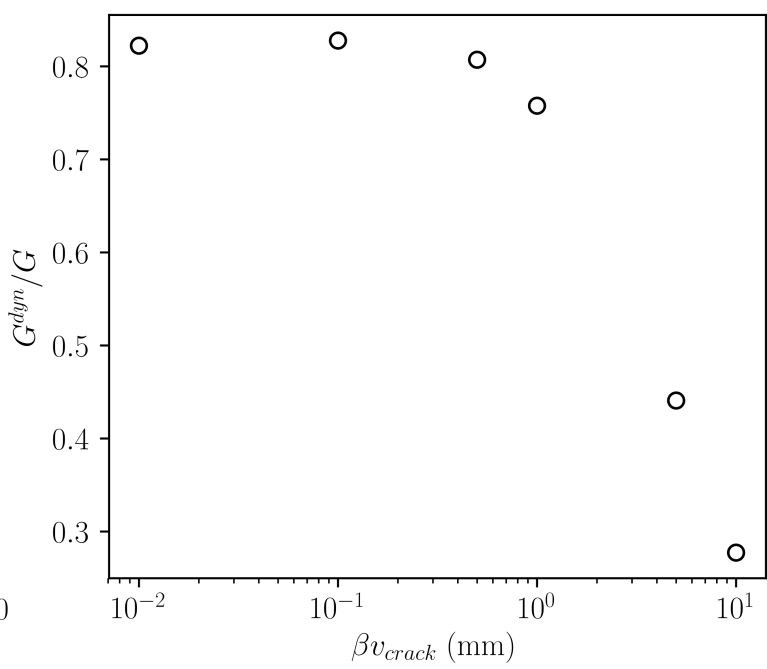

(b)

Figure 5: (a) Energy release rate as a function of crack length for several damping coefficients $\beta$ and (b) dynamic $\left(G^{d y n}\right)$ to quasi-static $(G)$ energy release rate ratio as a function of $\beta$ obtained for $v_{\text {crack }}=0.1 c_{R}$ crack velocity. 


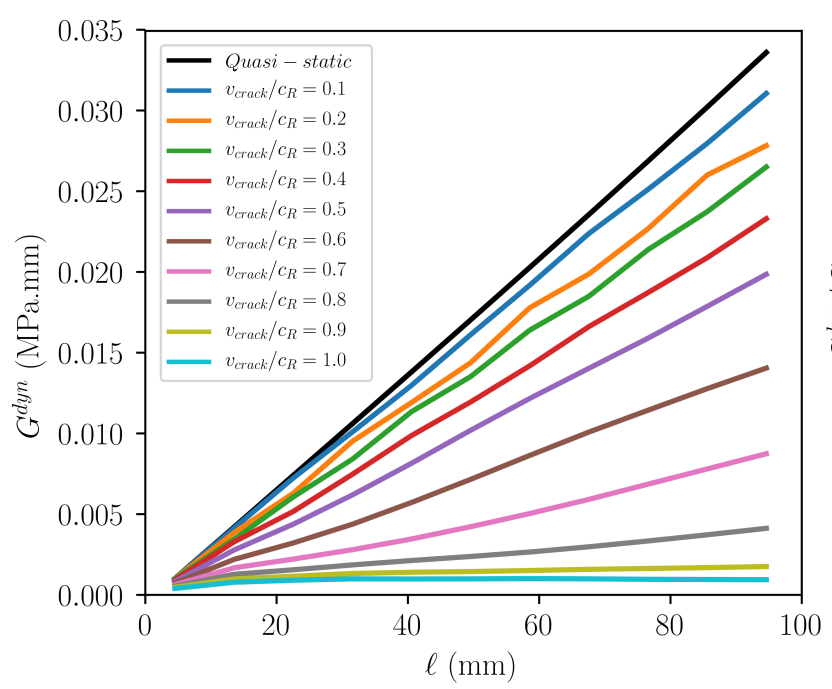

(a)

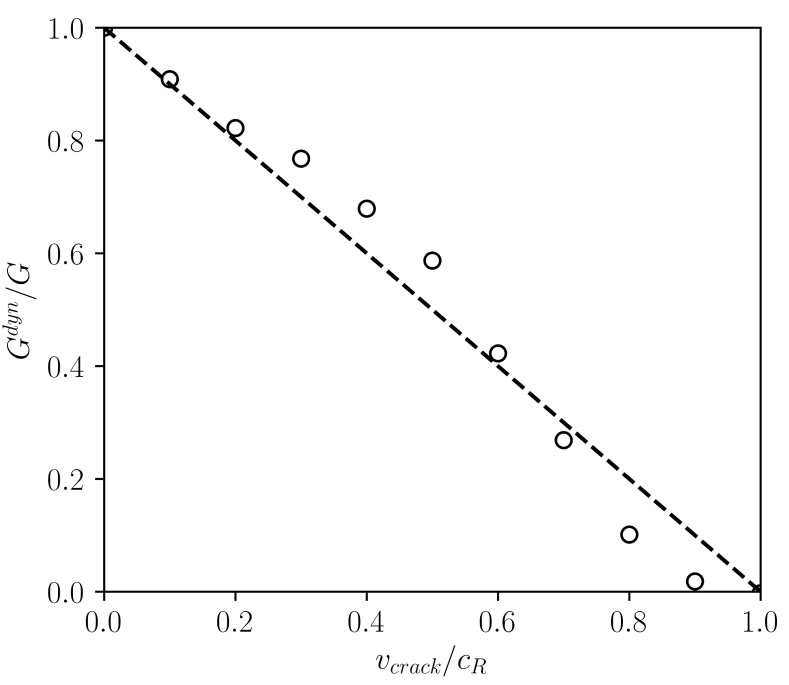

(b)

Figure 6: (a) Energy release rate as a function of crack length for several crack velocities. (b) Dynamic $\left(G^{d y n}\right)$ to quasi-static $(G)$ energy release rate ratio as a function of crack velocity to Rayleigh velocity ratio.

$$
J=\int_{\partial \Gamma}\left(W_{p} n_{x}(s)-\underline{\sigma} \cdot \mathbf{n}(s) \cdot \frac{\partial \mathbf{u}}{\partial x}\right) d s+\int_{\Gamma} \rho \frac{\partial^{2} \mathbf{u}}{\partial t^{2}} \cdot \frac{\partial \mathbf{u}}{\partial x} d x d y
$$

The integral is computed over an area $\Gamma$ around the crack tip delimited by a closed contour $\partial \Gamma$, $\mathrm{s}$ is the curvilinear abscissae along the contour and $\mathbf{n}$ is the contour normal unit vector pointing outwards. Fig. 7 shows the ERR variation as a function of the crack length computed using Eq. (18) for several crack velocities as well as the dynamic to quasi-static ERR ratios as a function

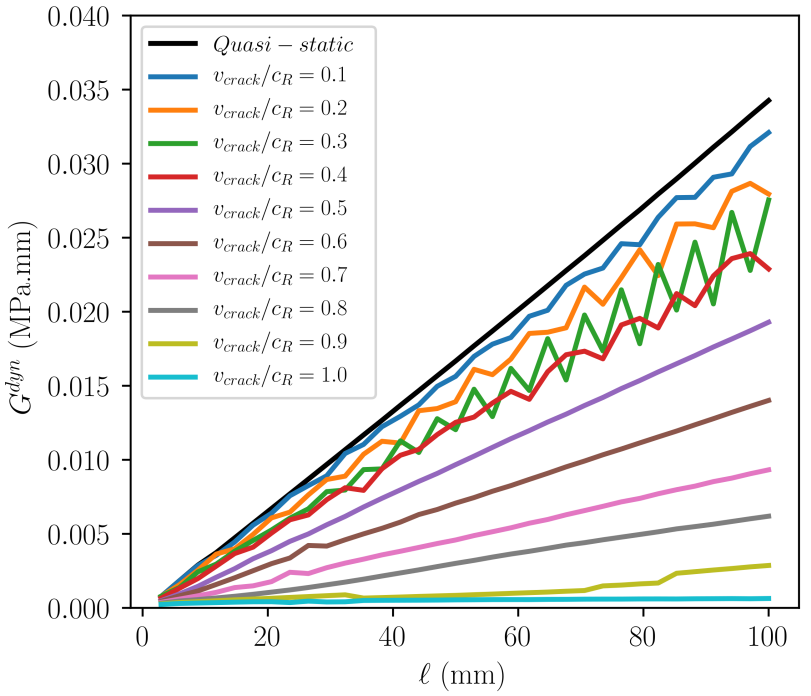

(a)

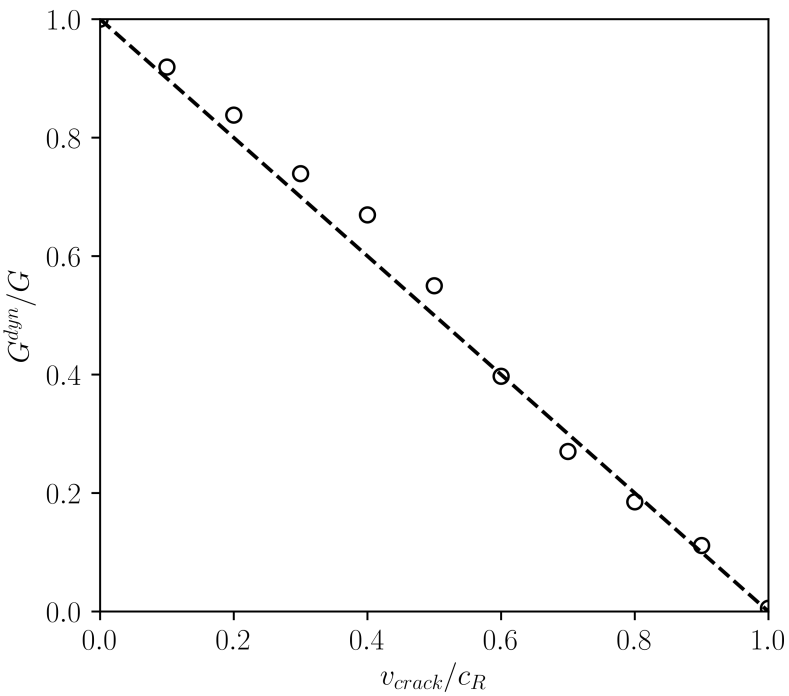

(b)

Figure 7: (a) Energy release rate as a function of crack length for several crack velocities. (b) Dynamic $\left(G^{d y n}\right)$ to quasi-static $(G)$ energy release rate ratio as a function of crack velocity to Rayleigh velocity ratio obtained using the dynamic J-integral (Eq. (18)). 
of the crack velocity normalized by the Rayleigh velocity.

The dynamic to quasi-static ERR ratio obtained using the dynamic J-integral exhibits a quasilinear variation as a function of the crack velocity to Rayleigh velocity ratio, similarly to that obtained previously from the energy balance.

\section{Transverse cracking in laminated composite}

We now study the $2 \mathrm{D}$ example of transverse cracking in the middle ply of $0^{\circ} / 90^{\circ} / 0^{\circ}$ laminated composite under tensile loading, which is a typical application of the CC $[5,52,53]$ for which failure is driven either by the stress (thick plies) or the energy (thin plies) criterion. Each ply exhibits a transversely isotropic behavior, the ply properties are given in Tab. $1[54,55,56]$ as well as the dimensions of the specimens, also depicted in Fig. 8. Dirichlet conditions are prescribed at both sides of the specimens and no debonding at the ply interface is considered. The studied range of ply thickness $(t)$ is $0.2 \mathrm{~mm}$ to $3 \mathrm{~mm}$. Since the stress is homogeneous within a ply, we consider that crack initiation occurs simultaneously in the whole ply thickness. A $t / 20$ mesh size is set in the vicinity of crack initiation location. The application of the CC quasi-static approach

\begin{tabular}{|c|c|c|c|c|}
\hline$E_{L}(\mathrm{GPa})$ & $E_{t}(\mathrm{GPa})$ & $\nu_{L T}$ & $\nu_{T T}$ & $\mu_{L T}(\mathrm{GPa})$ \\
\hline 127.3 & 9.2 & 0.3 & 0.4 & 4.8 \\
\hline \hline$\sigma_{c}(\mathrm{MPa})$ & $G_{c}\left(\mathrm{~J} / \mathrm{m}^{2}\right)$ & $\rho\left(\mathrm{kg} / \mathrm{m}^{3}\right)$ & $\mathrm{L}(\mathrm{mm})$ & $\mathrm{w}(\mathrm{mm})$ \\
\hline 63.9 & 248 & 1500 & 160 & 25 \\
\hline
\end{tabular}

Table 1: Properties of the ply and specimen dimensions (w denotes the specimen width in the out-of-plane direction).

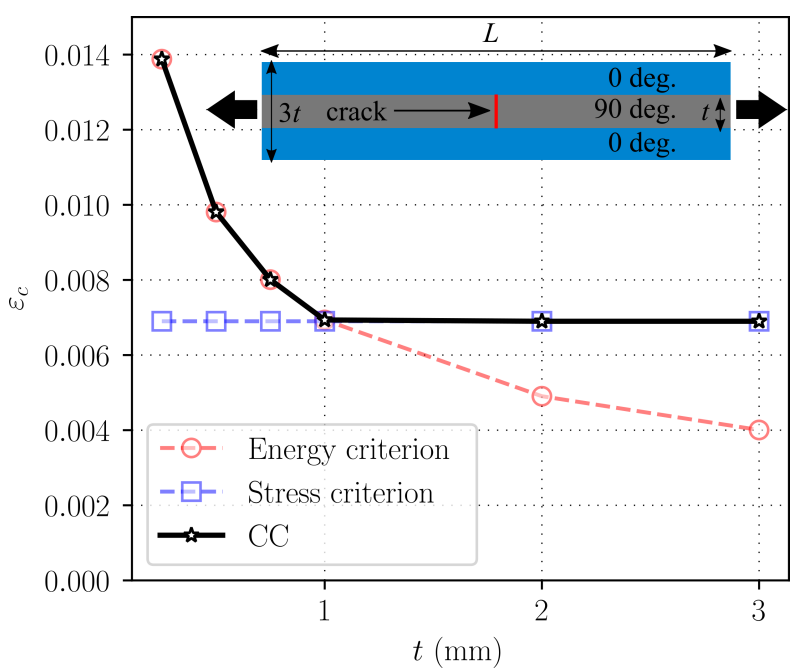

Figure 8: Initiation strain required to fulfill i) the stress, ii) the energy and iii) the coupled criterion as a function of the ply thickness obtained using the quasi-static CC approach. 
yields the following results (Fig. 8):

- For thick enough plies, the energy criterion is fulfilled for a loading smaller than that required to fulfill the stress criterion. Indeed, a sufficiently high amount of potential energy is stored and could be released for such a loading level, however since the stress criterion is not fulfilled, crack initiation can only occur for a loading level such that the stress in the ply attains the ply strength. Failure of thick plies is thus driven by the stress condition.

- For thin enough plies, larger loading levels than for thick plies are required in order to fulfill the energy criterion because less energy is stored and thus the potential energy release due to transverse crack initiation is smaller. Therefore, contrary to thicker plies, crack initiation cannot occur when the ply stress reaches the ply strength but the loading level has to be increased so that the energy criterion is also fulfilled. Failure of thin plies is thus driven by the energy condition.

We investigate the $\mathrm{CC}$ dynamic application in the case of transverse cracking in laminate. The same model as for the CC quasi-static application is employed except that crack initiation occurs during a time period $\Delta t_{0}$ so that the crack velocity $v_{\text {crack }}$ is defined as the ratio between the ply thickness and the initiation time $\Delta t_{0}$. Fig. 9a-b shows the potential and kinetic energy variations as a function of the crack length for several crack velocities. It can be observed that a larger crack velocity induces a smaller potential energy release as well as larger kinetic energy creation. Therefore, the larger the crack velocity, the smaller the IERR (Fig. 9c). Moreover, it can be observed that the potential and kinetic energies, and thus the ERR, tend towards the quasi-static

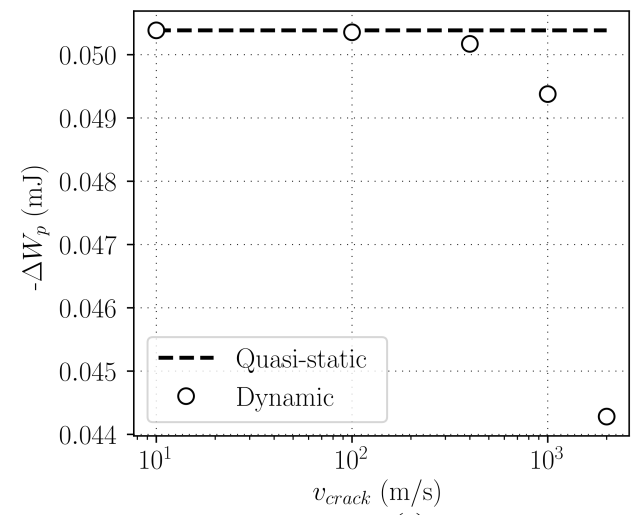

(a)

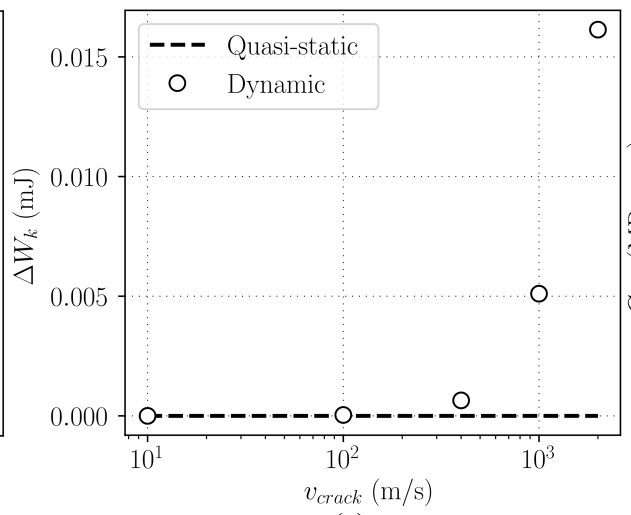

(b)

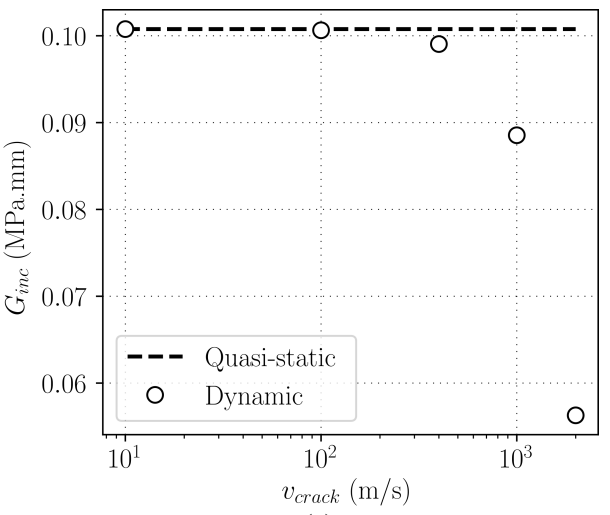

(c)

Figure 9: (a) Potential energy release, (b) kinetic energy variation and (c) incremental energy release rate as a function of the crack velocity. 
values when the crack velocity tends towards 0 .

Fig. 10 depicts the CC solution for several crack velocities. Fig. 10a-b shows the strain that must be prescribed in order to fulfill either the stress or the energy criterion for several crack velocities in the case of a thick (Fig. 10a) or a thin (Fig. 10b) ply. It can first be noted that the stress criterion does not depend on the crack velocity since it is computed prior to crack initiation and that the loading is quasi-static.

Whatever the ply size, it can be observed that the larger the crack velocity, the larger the loading level required in order to fulfill the energy criterion. Therefore, the initiation loading level is larger than in the quasi-static case for thin plies since failure is driven by the energy criterion. On the contrary, for thick plies failure is driven by the stress criterion in the quasi-static case. The increase in the loading level required to fulfill the energy criterion leads to two possibilities:

- case 1) If the crack velocity remains sufficiently small, the increase in the loading level required to fulfill the energy criterion is not sufficient so that it overcomes the loading levels required to fulfill the stress criterion. Thus, failure remains driven by the stress criterion and occurs at the same loading level than the one obtained using the CC quasi-static approach.

- case 2) For large enough crack velocities $\left(v_{\text {crack }}>2000 \mathrm{~m} / \mathrm{s}\right.$ for this example), the increase in the loading level required to fulfill the energy criterion is sufficiently large so that it overcomes the loading level required to fulfill the stress criterion. This situation would lead to failure being driven by the energy criterion even in the case of thick plies.

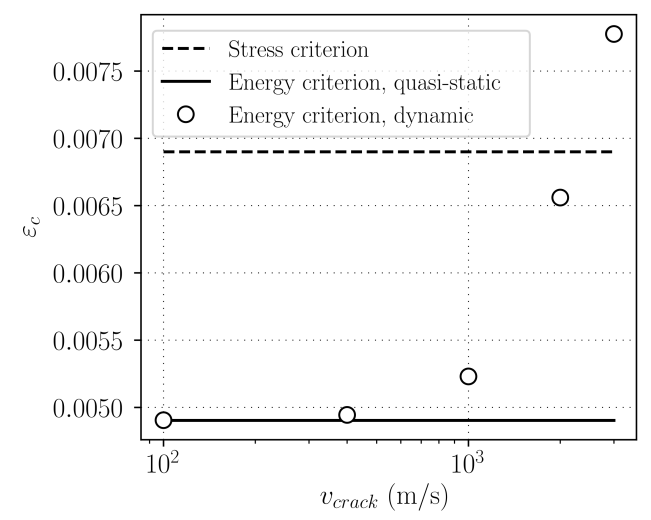

(a)

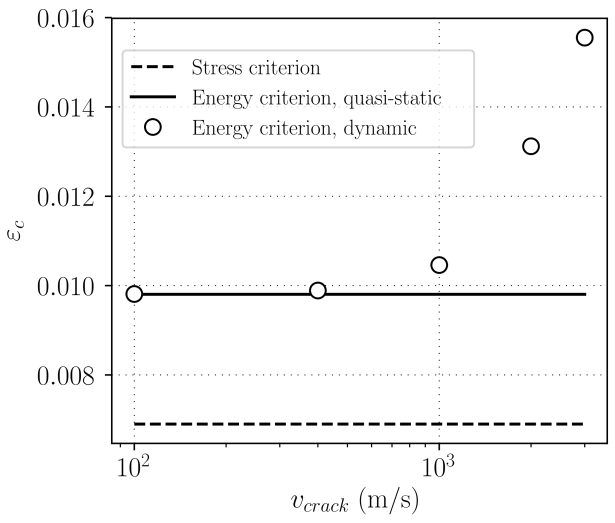

(b)

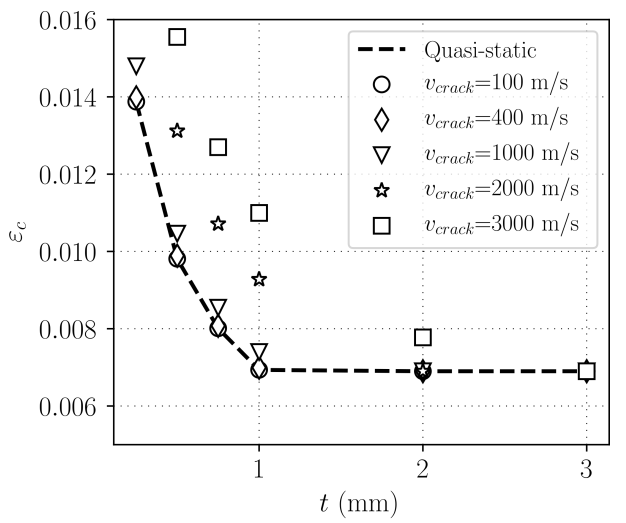

(c)

Figure 10: Prescribed strain required to fulfill the stress or the energy criterion as a function of the crack velocity in the case of a (a) thick or (b) thin ply. (c) Initiation strain as a function of the ply thickness for several crack velocities. 
Fig. 10c shows the initiation strain as a function of the ply thickness obtained using either the CC quasi-static or dynamic approaches. If crack velocity is sufficiently small, a transition between failure driven by the stress (for thick plies) or the energy (for thin plies) criterion is observed. Whereas the loading levels are similar to the ones obtained using the CC quasi-static approach for thick plies, the loading level variation as a function of the ply thickness depends on the crack velocity for thin plies. For sufficiently large crack velocities, the transition between failure driven by the stress or the energy criterion would no longer be observed and failure would only be driven by the energy criterion.

\section{Circular hole specimen under tensile loading}

The CC dynamic approach under quasi-static tensile loading is illustrated by studying holed PMMA specimens failure under tensile loading [57] (Fig. 11). The material properties are the following: $E=3000 \mathrm{MPa}, \nu=0.37, \rho=1000 \mathrm{~kg} / \mathrm{m}^{3}, \sigma_{c}=72 \mathrm{MPa}$ and $G_{c}=290 \mathrm{~J} / \mathrm{m}^{2}$. Specimens (length: $100 \mathrm{~mm}$, section: 10x30 $\left.\mathrm{mm}^{2}\right)$ with several hole radii $(r=0.3,0.6,1$ and $1.5 \mathrm{~mm})$ were tested under uniaxial tensile loading. For this example, the prescribed stresses at crack initiation predicted using the quasi-static approach of the $\mathrm{CC}$ underestimated the initiation stresses obtained experimentally [57, 58]. Li and Zhang [57] proposed to enrich the CC with a third parameter representing the roughness of the fracture pattern and thus obtained a better agreement with experimental results. Leite et al. [11] performed tensile tests in PMMA specimens containing a circular hole and observed crack initiation using a high speed camera to measure a $700 \mathrm{~m} / \mathrm{s}$ average velocity. We evaluate herein the influence of considering dynamic instead of quasi-static CC to predict crack initiation in such a configuration.

Based on recommendations derived for the CC quasi-static approach $[14,59]$, a $5 \mu$ m minimum

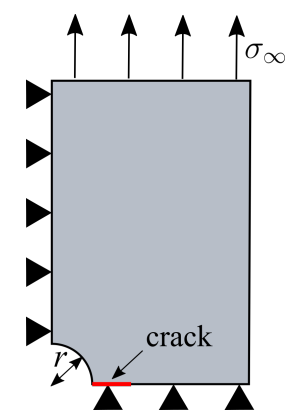

Figure 11: Specimen geometry and boundary conditions for circular hole specimen under tensile loading. 
mesh size is set near the hole, the mesh being unrefined far from the hole, typically resulting in a model with around 10000 degrees of freedom. The characteristic material length $l_{m a t}=\frac{E G_{c}}{\left(1-\nu^{2}\right) \sigma_{c}^{2}}$ is $194 \mu \mathrm{m}$ so that the minimum mesh size is $\approx \frac{1}{40} l_{\text {mat }}$, which is sufficiently small to capture the initiation crack length and thus properly solve Eq. (4).

The CC dynamic approach under quasi-static loading requires the calculation of the stress and the energy criteria. The former only requires a linear elastic calculation, similarly to the quasistatic approach. Since the stress variation is computed on the presupposed crack path prior to crack initiation, it does not depend on the crack velocity. However, the crack velocity profile must be taken into account to assess the energy criterion. For the sake of simplicity, we first assume a constant crack velocity profile. The nodes along the crack path are unbuttoned following the prescribed crack velocity, which enables the calculation of the potential and kinetic energies as a function of the crack length. In the following, we illustrate the CC solution for a specimen with $r=0.15 \mathrm{~mm}$ hole radius, the results being qualitatively the same for other hole radii. Fig. 12a-b shows the potential and kinetic energy variations as a function of the crack length for several crack velocities. It can be observed that a larger crack velocity induces a smaller potential energy release as well as larger kinetic energy creation. Therefore, for a given crack length, the larger the crack velocity, the smaller the IERR (Fig. 12). Moreover, it can be observed that the IERR tends towards the quasi-static value when the crack velocity tends towards 0 .

Fig. 13 shows the CC solution for several crack velocities. Fig. 13a shows the displacement that must be prescribed in order to fulfill either the stress or the energy criterion for several crack velocities. It can be observed that, for a given crack length, the larger the crack velocity the larger

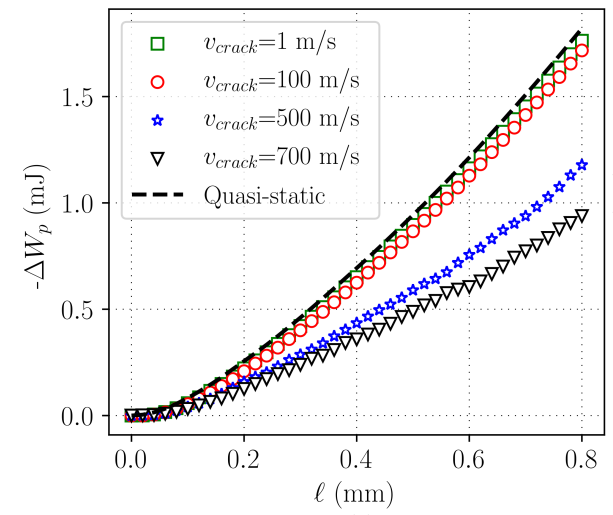

(a)

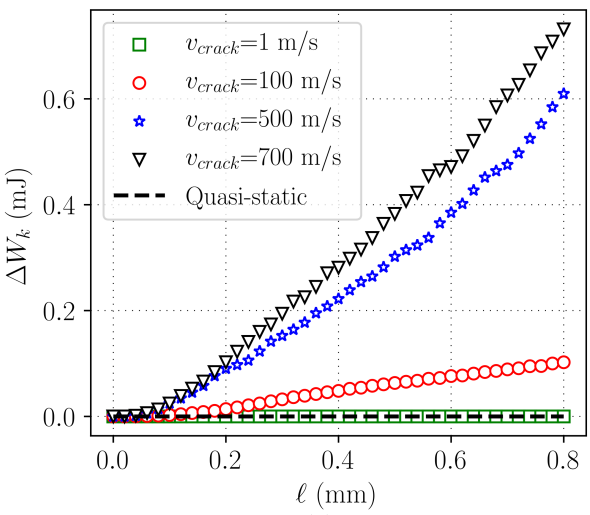

(b)

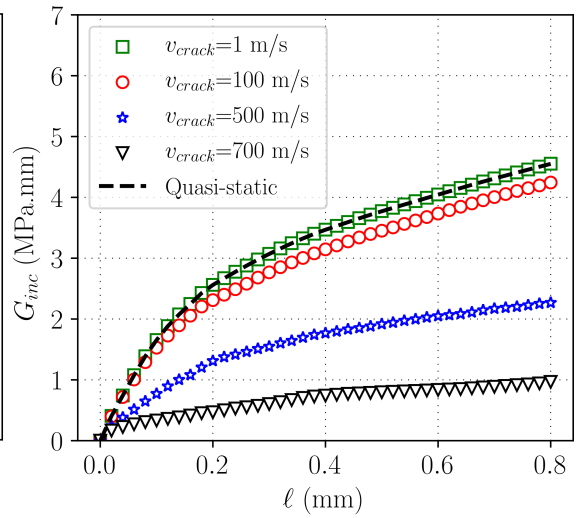

(c)

Figure 12: (a) Potential energy release, (b) kinetic energy variation and (c) incremental dynamic energy release rate as a function of the crack length for several crack velocities. 


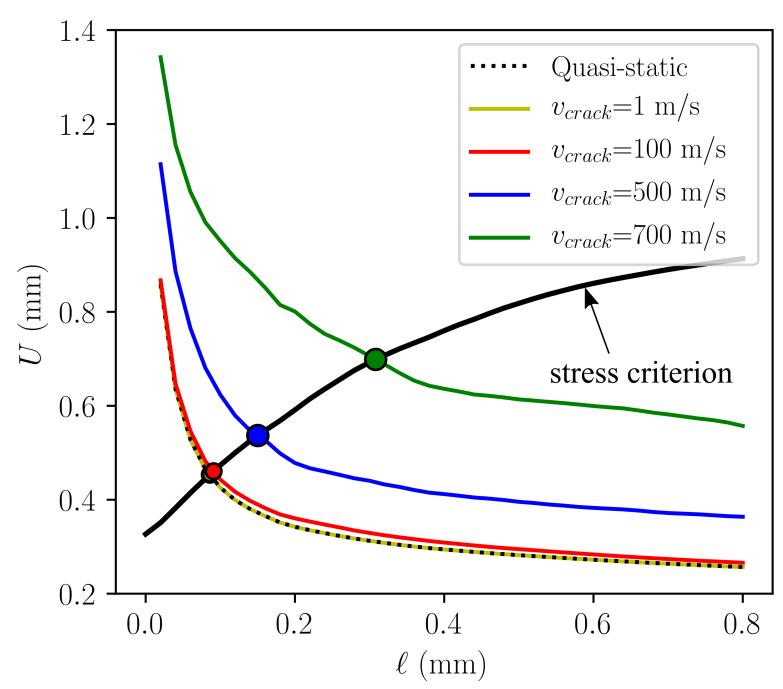

(a)

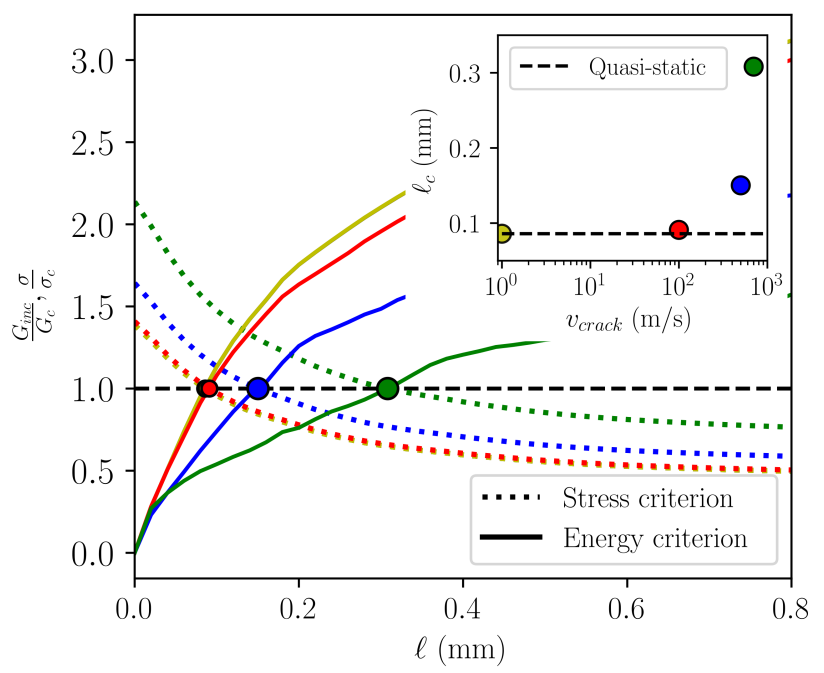

(b)

Figure 13: (a) Prescribed displacement required to fulfill the stress (dashed line) or the energy (solid lines) condition and (b) stress and energy criteria as a function of the crack length for several crack velocities. The curves corresponding to quasi-static condition is superimposed with the curves obtained for $v_{\text {crack }}=1 \mathrm{~m} / \mathrm{s}$

the prescribed displacement required to fulfill the energy criterion. On the other hand, the stress criterion is reached at the same prescribed loading level for a given crack length since it does not depend on the crack velocity. Therefore, the initiation displacement, determined as the minimum displacement for which both criteria are simultaneously fulfilled, increases with increasing crack velocity.

Accordingly, the initiation crack length also increases with increasing crack velocity, which is also highlighted by displaying stress and energy criteria as a function of the crack length (Fig. 13b).It can also be noted that the initiation displacement and crack length tend towards the values obtained using the CC quasi-static approach when the crack velocity tends towards 0 .

Fig. 14 shows the prescribed stress as a function of the hole radius obtained experimentally or numerically using the either the CC quasi-static or dynamic approach for several crack velocities. As explained previously, the CC quasi-static approach underestimates the initiation prescribed stress compared to experimental measurements. For a given hole radius, the larger the crack velocity the larger the initiation stress. It can thus be observed that considering dynamic effects during crack initiation provides initiation stresses larger than for the quasi-static approach. In particular, the initiation stress variation measured experimentally is well reproduced numerically for a $v_{\text {crack }}=700 \mathrm{~m} / \mathrm{s}$ crack velocity. This crack velocity is in the order of magnitude of experimentally measured crack velocities in PMMA [11, 60, 61, 62, 63]. 


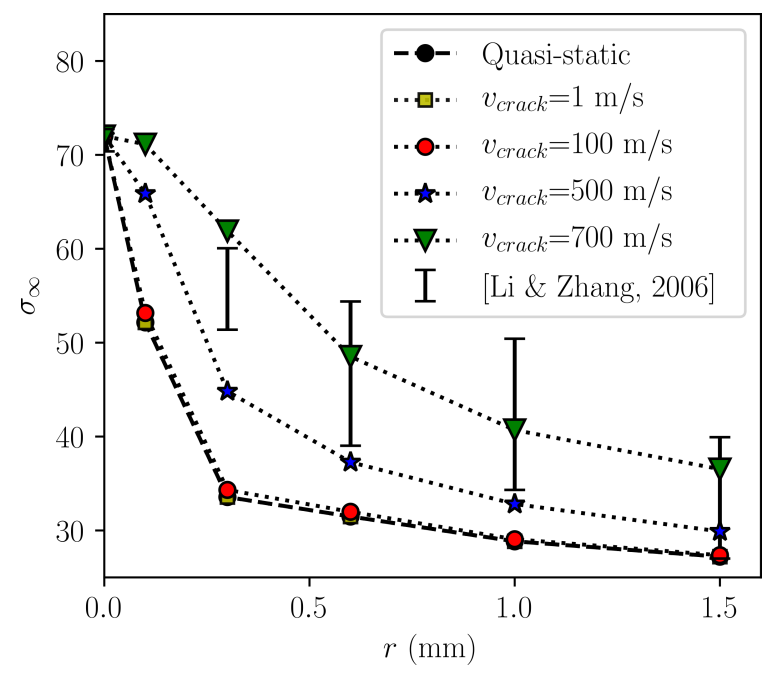

Figure 14: Prescribed stress at crack initiation as a function of the specimen hole diameter obtained experimentally and numerically using the $\mathrm{CC}$ dynamic approach for different mean crack velocities.

\section{Compression of circular hole specimen}

In this section we assess the influence of the crack velocity profile on crack initiation under quasistatic imposed displacement $U_{0}$. We study crack initiation in a drilled specimen under compression (Fig. 15). The circular hole diameter is $5 \mathrm{~mm}$, the specimen dimensions are $w=20 \mathrm{~mm}, h=30 \mathrm{~mm}$.

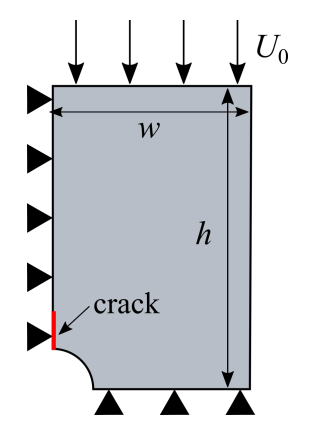

Figure 15: Specimen geometry and dimensions and boundary conditions for drilled hole specimen under compression.

The material properties are $E=3000 \mathrm{MPa}, \nu=0.37, \rho=1000 \mathrm{~kg} / \mathrm{m}^{3}, \sigma_{c}=47 \mathrm{MPa}$ and $G_{c}=$ $500 \mathrm{~J} / \mathrm{m}^{2}$. The characteristic material length $l_{\text {mat }}=\frac{E G_{c}}{\left(1-\nu^{2}\right) \sigma_{c}^{2}}$ is $787 \mu \mathrm{m}$ and the initiation length obtained using the quasi-static CC solution is $300 \mu \mathrm{m}$. The minimum mesh size near the hole is $\approx \frac{l_{m a t}}{80}$, which is sufficiently small to capture the initiation crack length and thus properly solve Eq. (4). We consider several mean crack initiation velocities, i.e. $v_{0}=100 \mathrm{~m} / \mathrm{s}\left(\approx 0.1 c_{R}\right)$, $v_{0}=300 \mathrm{~m} / \mathrm{s}\left(\approx 0.3 c_{R}\right)$ and $v_{0}=500 \mathrm{~m} / \mathrm{s}\left(\approx 0.5 c_{R}\right)$ as well as consider four different velocity profiles, namely: 
- $v_{\text {crack }}(t)=v_{0}($ constant velocity profile)

- $v_{\text {crack }}(t)=2 v_{0} \frac{t}{t_{0}}$ (linear velocity profile)

- $v_{\text {crack }}(t)=\frac{3 v_{0}}{2} \sqrt{\frac{t}{t_{0}}}($ square root $(\mathrm{SQRT})$ velocity profile)

- $v_{\text {crack }}(t)=\frac{v_{0}}{2} \sqrt{\frac{t_{0}}{t}}$ (inverse square root (ISQRT) velocity profile)

All these velocity profiles, depicted in Fig. 16 for a $v_{0}=100 \mathrm{~m} / \mathrm{s}$ mean crack velocity, share the property of having the same $v_{0}$ mean crack velocity during the initiation time $t_{0}$. Figs. 17-19(a-b) show the potential and kinetic energy variations as a function of the crack length for the different crack velocity profiles, for respectively $v_{0}=100 \mathrm{~m} / \mathrm{s}$ (Fig. 17), $v_{0}=300 \mathrm{~m} / \mathrm{s}$ (Fig. 18) and $v_{0}=500 \mathrm{~m} / \mathrm{s}$ (Fig. 19) mean crack velocities.

It can be observed that for sufficiently small mean crack velocities, the potential and kinetic energy variations exhibit relatively small differences whatever the crack velocity profile. It can be noted that for a given crack length, the potential energy release and kinetic energy creation are larger (respectively smaller) if the instantaneous crack velocity is larger (respectively smaller). As a consequence, no major differences are observed on the IERR, except for the ISQRT velocity profile, for which the IERR is slightly smaller for small crack lengths (Fig. 17c). This is related to the fact that for small crack lengths, the crack velocity for ISQRT profile is large (larger than the mean crack velocity) whereas it starts from zero for linear and SQRT profiles. The larger

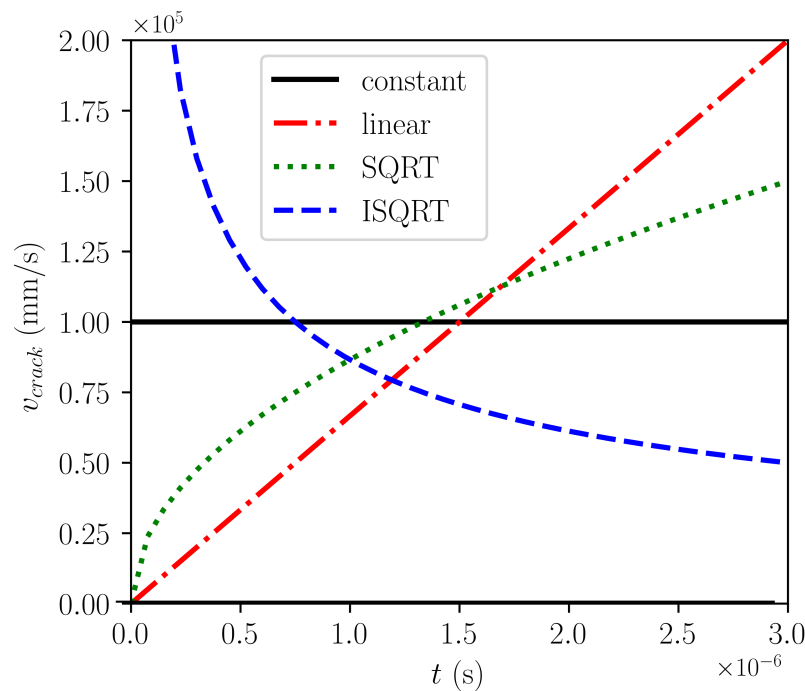

(a)

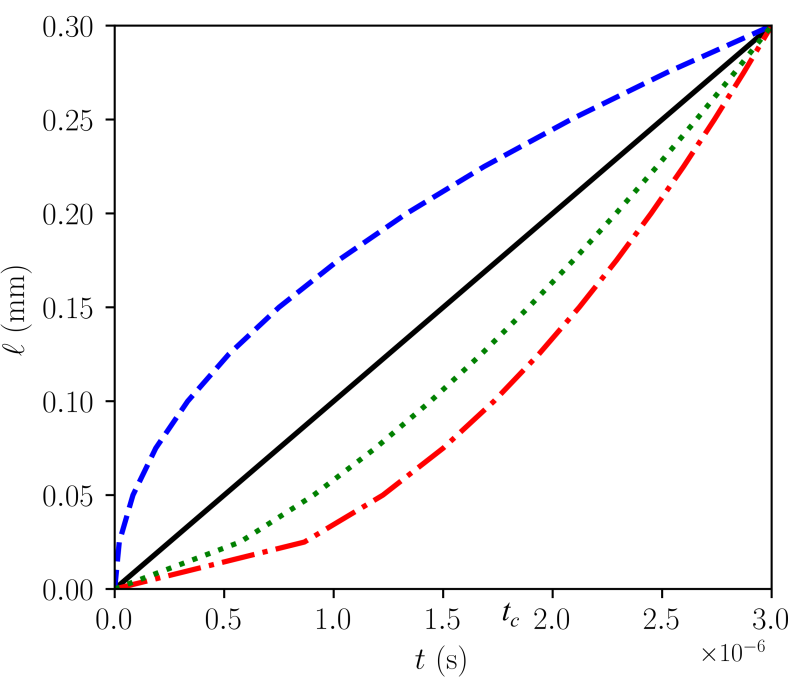

(b)

Figure 16: Crack (a) velocity and (b) corresponding length profiles as a function of time for a $v_{0}=100 \mathrm{~m} / \mathrm{s}$ mean crack velocity. 


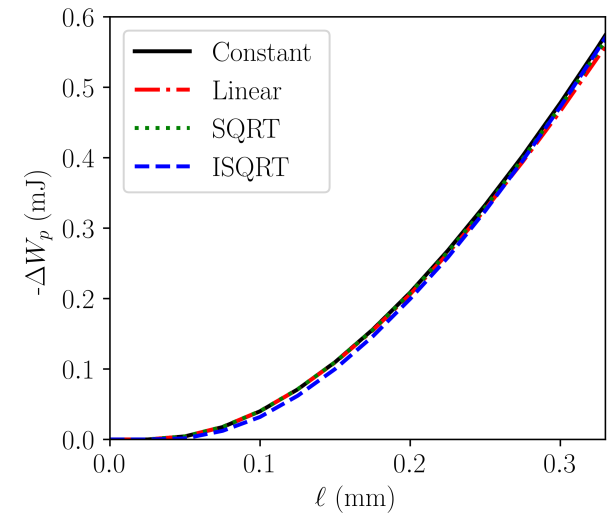

(a)

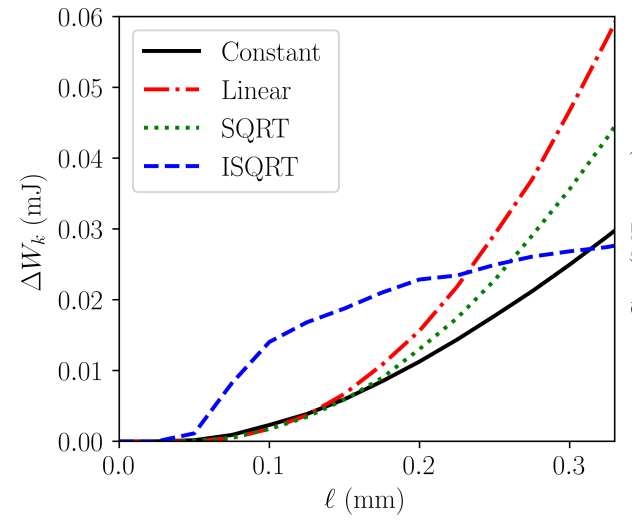

(b)

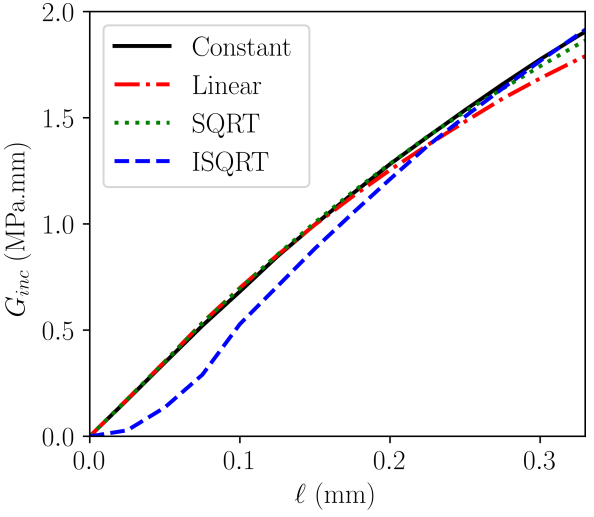

(c)

Figure 17: (a) Potential energy release, (b) kinetic energy variation and (c) incremental dynamic energy release rate as a function of the crack length obtained with the different crack velocity profiles depicted in Fig. 16 for a $v_{0}=100 \mathrm{~m} / \mathrm{s}$ mean crack velocity.

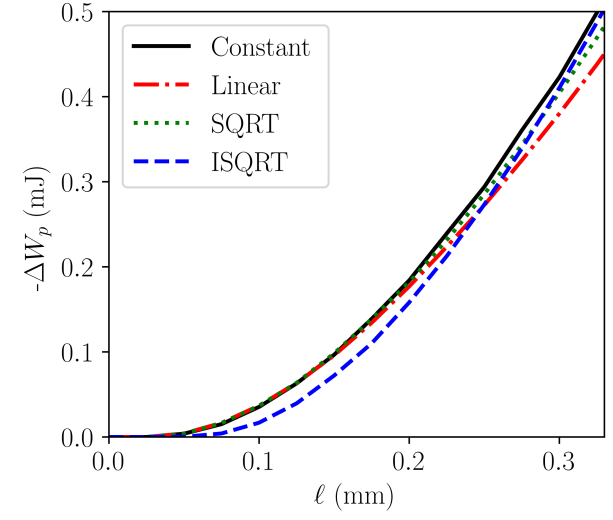

(a)

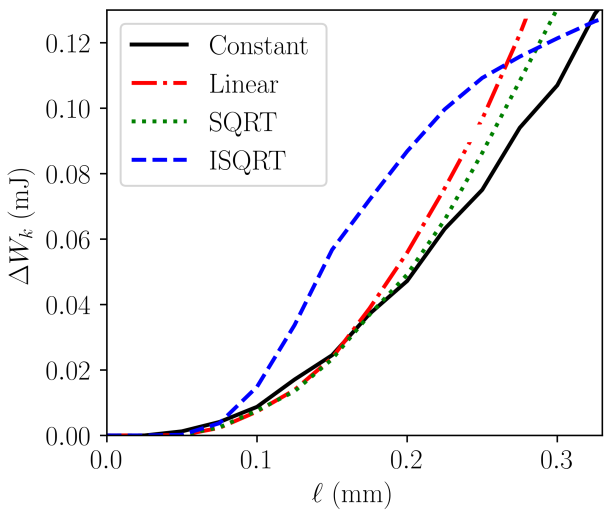

(b)

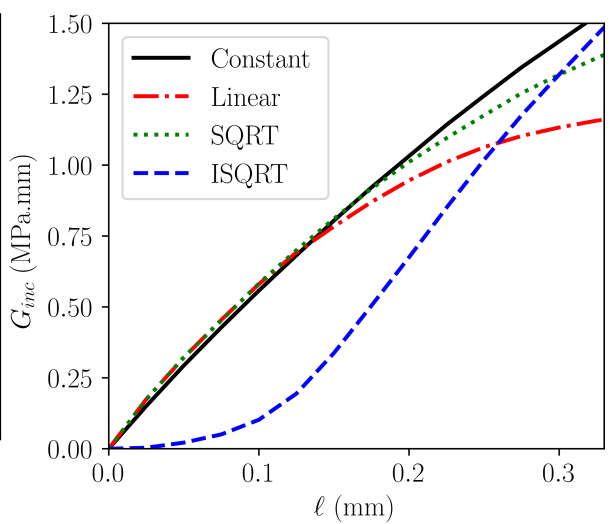

(c)

Figure 18: (a) Potential energy release, (b) kinetic energy variation and (c) incremental energy release rate as a function of the crack length obtained with the different crack velocity profiles depicted in Fig. 16 for a $v_{0}=300 \mathrm{~m} / \mathrm{s}$ mean crack velocity.

the mean crack velocity, the larger the difference in potential and kinetic energies and thus the larger the difference in the IERR between the different profiles (Figs. 18-19c). This has a direct consequence on the predicted initiation crack length and prescribed displacement. Fig. 20 shows the displacement that must be prescribed in order to fulfill either the stress or the energy criterion for the different crack velocity profiles and for different mean crack velocities. Since the loading is quasi-static, the same prescribed displacement variation as a function of the crack length obtained using the stress condition is obtained for all velocity profiles. However, the displacement that must be prescribed in order to fulfill the energy condition differs for the different profiles. It yields a $1.5 \%$ difference for $v_{0}=100 \mathrm{~m} / \mathrm{s}$ mean crack velocity between the prescribed displacement obtained for the different profile, this difference increases up to respectively $18 \%$ and $54 \%$ for $v_{0}=300 \mathrm{~m} / \mathrm{s}$ and 


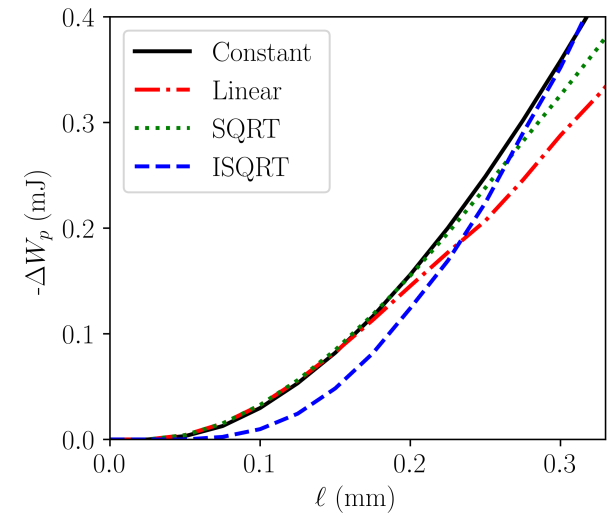

(a)

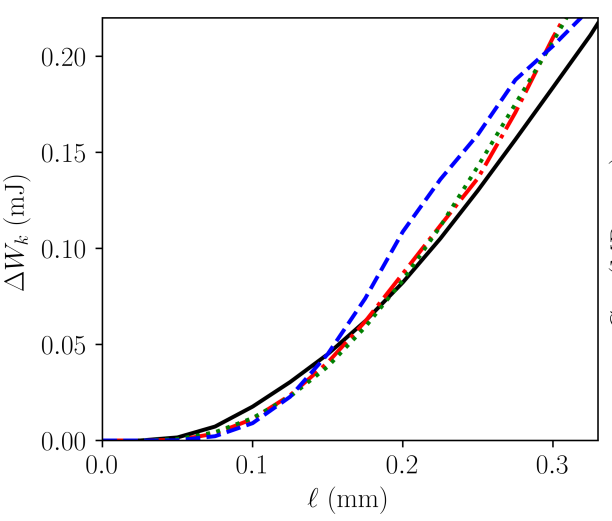

(b)

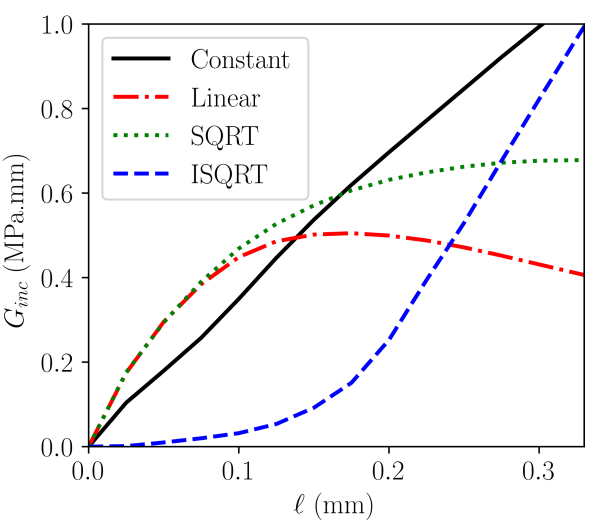

(c)

Figure 19: (a) Potential energy release, (b) kinetic energy variation and (c) incremental dynamic energy release rate as a function of the crack length obtained with the different crack velocity profiles depicted in Fig. 16 for a $v_{0}=500 \mathrm{~m} / \mathrm{s}$ mean crack velocity.

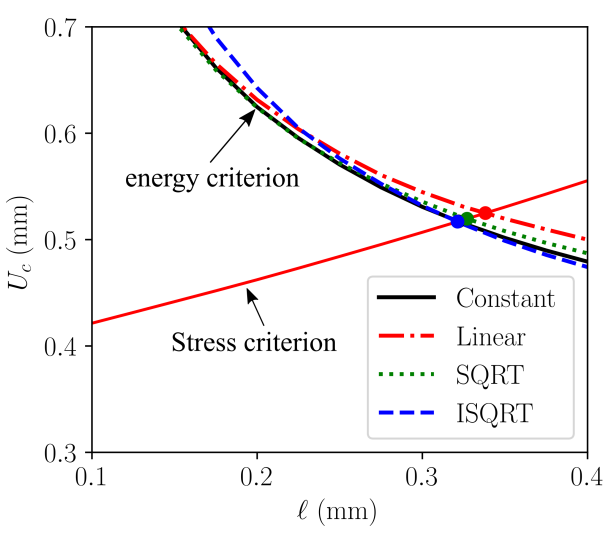

(a)

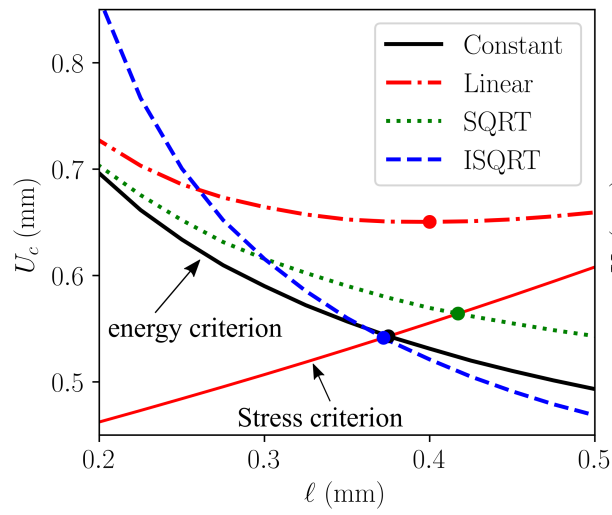

(b)

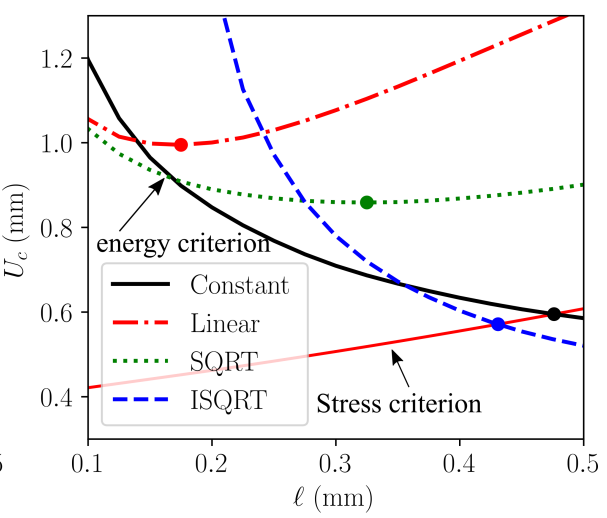

(c)

Figure 20: Prescribed displacement required to fulfill the stress or the energy conditions as a function of the crack length for the different crack velocity profiles depicted in Fig. 16 for a) $\left.v_{0}=100 \mathrm{~m} / \mathrm{s}, \mathrm{b}\right) v_{0}=300 \mathrm{~m} / \mathrm{s}$ and c) $v_{0}=500 \mathrm{~m} / \mathrm{s}$ mean crack velocities.

$v_{0}=500 \mathrm{~m} / \mathrm{s}$ mean crack velocities.

It finally appears that the crack velocity profile during initiation has a small influence on the crack length and loading level obtained with the $\mathrm{CC}$ only for small mean crack velocities. In this case, a constant crack velocity profile may be employed to assess dynamic crack initiation. However, larger differences are obtained for larger crack velocities, for which not only the mean crack velocity but also the crack velocity profile has to be determined experimentally, which could be estimated using a rapid camera to observe crack initiation. 


\section{Time-dependent loading}

In this last example, the dynamic approach is evaluated in the case of a time-dependent loading. The studied example is crack initiation in the vicinity of a V-notch subjected to a stress wave. The example is close to that studied by Kishimoto et al. [51] except that the initial crack is herein replaced by a V-notch (Fig. 21). The specimen dimensions are $L=52 \mathrm{~mm}, H=20 \mathrm{~mm}, a=12 \mathrm{~mm}$

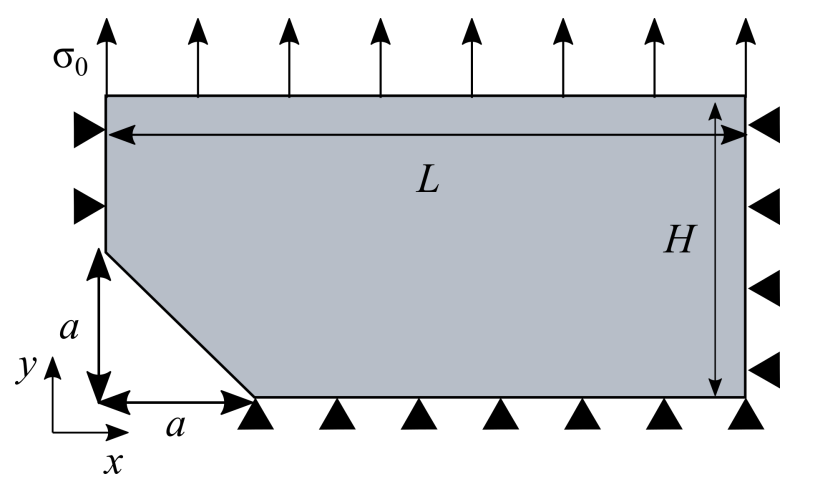

Figure 21: Geometry and V-notch specimen dimensions for the stress wave loading.

and a $0.1 \mathrm{~mm}$ mesh size is prescribed in the whole model. The material properties are $E=73.5 \mathrm{GPa}$, $\nu=0.25, \rho=2450 \mathrm{~kg} / \mathrm{m}^{3}$, which results in a $c_{R}=3179 \mathrm{~m} / \mathrm{s}$ Rayleigh wave speed. The calculation are performed assuming a constant crack velocity profile $\left(v_{\text {crack }}=0.4 c_{R}\right)$ during initiation. The material fracture properties are set to $\sigma_{c}=250 \mathrm{MPa}$ and $G_{c}=1000 \mathrm{~J} / \mathrm{m}^{2}$. The prescribed loading is a constant stress level $\sigma_{0}=100 \mathrm{MPa}$ on the upper edge. Under such a loading, a stress wave emanates and propagates from the upper edge. Therefore, the stress field now depends on time, and especially the opening stress along the $\mathrm{V}$-notch bisector $\sigma_{y y}$, which is null at the beginning of the calculation and increases as the stress wave front reaches the V-notch tip vicinity. As explained in Section 2, the stress criterion has to be checked at every time step in order to determine the admissible crack lengths corresponding to a stress level larger than the material strength. The stress variation along the $\mathrm{V}$-notch bisector as a function of the distance to the V-notch tip is shown in Fig. 22a for several time steps. It can be observed that for a given distance to the V-notch tip, the stress increases with increasing time. Therefore, the range of admissible crack lengths obtained based on the stress condition also increases with increasing time ( $c f$. inset of Fig. 22a). Based on the set of admissible crack lengths obtained from the stress condition, it is possible to compute the energy condition only for the determined range of admissible crack lengths for a given time increment, which saves computational time. In the present example however, for the 


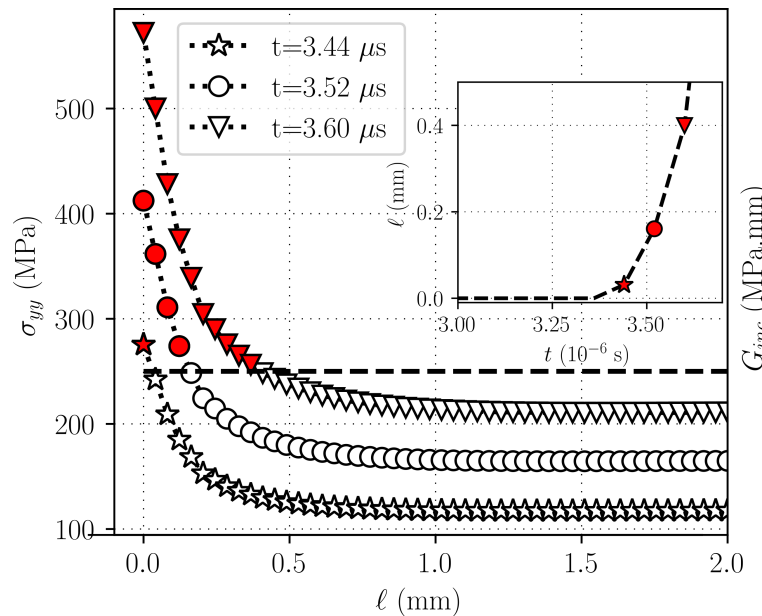

(a)

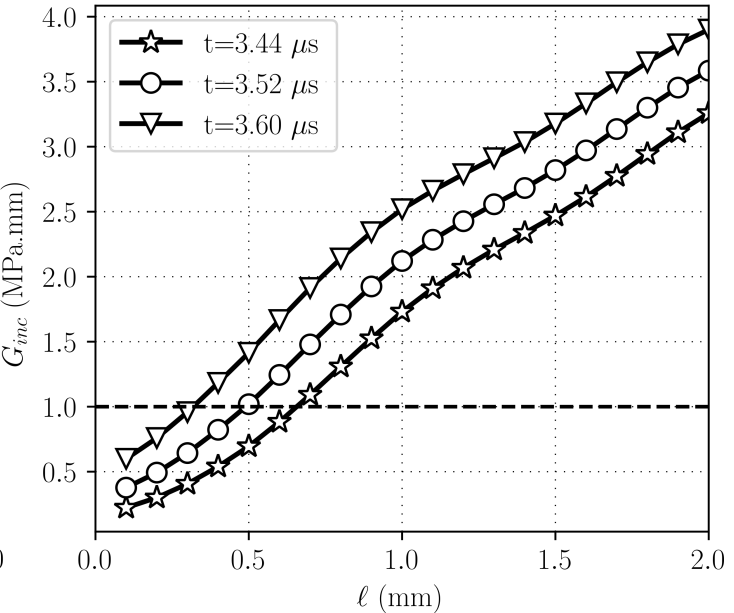

(b)

Figure 22: (a) Stress variation along the V-notch bisector prior to crack initiation and (b) incremental energy release rate as a function of the crack length.

sake of clarity, we computed the IERR for a larger range of crack lengths to illustrate the dynamic $\mathrm{CC}$ approach solution. It is depicted in Fig. 22b as a function of the crack length for several time steps. With increasing time, the IERR increases for a given crack length, thus the range of admissible crack lengths increases with increasing time. The initiation time and crack length can thus be determined as the minimum time for which a non-empty intersection of the admissible crack length sets obtained with the stress and the energy criteria is obtained. The stress and energy criteria are depicted in Fig. 23 for several time increments. For $t=3.44 \mu \mathrm{s}$ and $t=3.52 \mu \mathrm{s}$, it can be

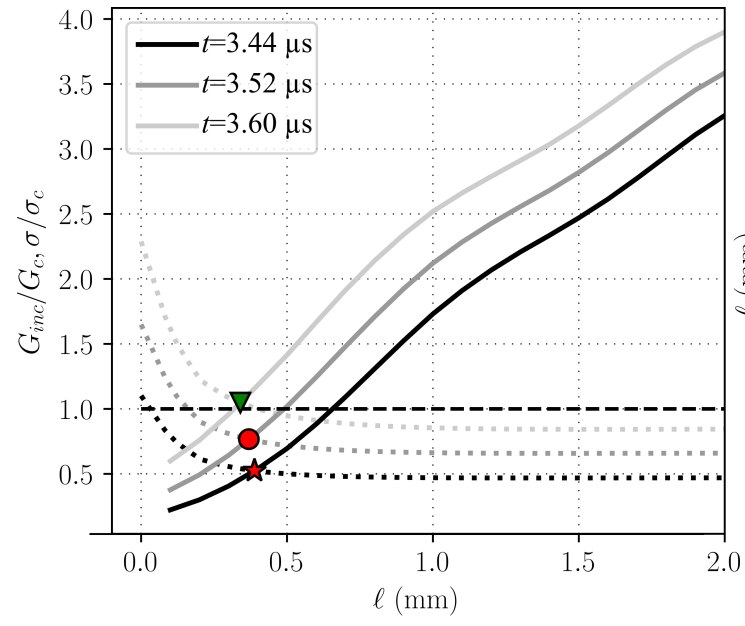

(a)

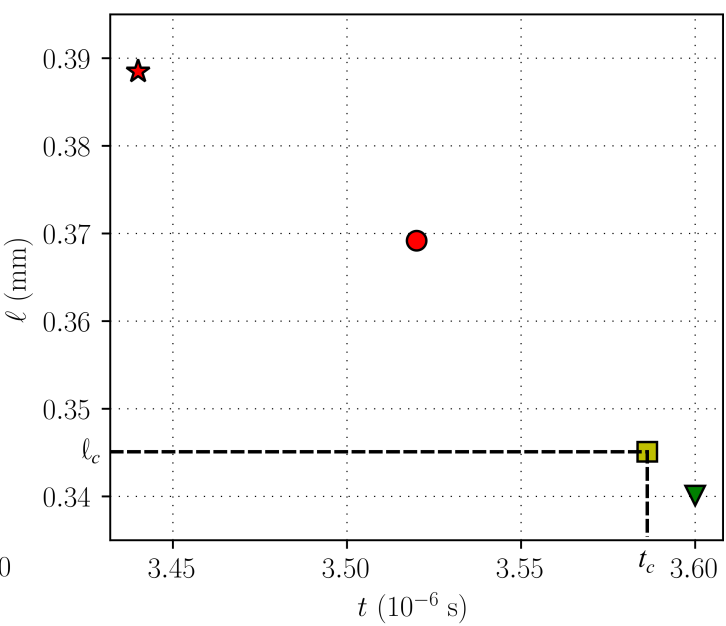

(b)

Figure 23: (a) Stress and energy criteria as a function of crack length for several time increments and (b) crack length for which both curves cross as a function of time. The initiation time is the minimum time for which both criteria are simulatenously met. 
observed that there are no crack lengths for which both criteria are simultaneously fulfilled, so that crack initiation cannot occur. On the contrary, a solution is found at time $t=3.60 \mu \mathrm{s}$, it is even observed that the criteria are overfulfilled, which indicates that crack initiation time lies within $t=3.52 \mu \mathrm{s}$ and $t=3.60 \mu \mathrm{s}$. The initiation time $t_{c}$ and crack length $l_{c}$ can be thus determined by interpolation of the computed values so that both criteria are simultaneously strictly fulfilled (Fig. 23b). Finally, the dynamic CC approach under time-dependent loading requires more calculations since the stress and energy conditions have to be calculated for several time increments instead of only once in the case of a quasi-static loading.

\section{Conclusion}

We extend the coupled criterion of Finite Fracture Mechanics in order to take into account dynamic effects occurring during crack initiation. Compared to its original form dedicated to study quasi-static configurations, the main change lies in accounting for the crack following a given velocity profile during initiation. Accordingly, the kinetic energy variation due to crack initiation cannot be neglected and is taken into account in the energy balance from which the energy condition of the $\mathrm{CC}$ is derived.

The proposed extension of the CC can be applied to either quasi-static or dynamic loading configurations. The former only requires several calculations with varying crack lengths following a prescribed velocity profile, whereas these calculations have to be performed for different time steps for the latter. Indeed, the stress and the energy conditions must be checked for several time increments (or loading levels) and the initiation time and crack length are determined as the minimum time for which both conditions are fulfilled.

Under either quasi-static or dynamic loading conditions, the dynamic CC approach predicts a crack jump at initiation, which results from the simultaneous fulfillment of both conditions, similarly to the quasi-static approach. However, in the dynamic approach, this crack jumps occurs during a given time that depends on the crack velocity. Taking into account the crack propagation following a certain velocity profile leads to a decrease in the potential energy release compared to the quasi-static case, as well as an increase in kinetic energy. It results in a decrease of the IERR and thus crack initiation at a larger loading level compared to the quasi-static approach. The larger the crack velocity, the larger the initiation loading and crack length. Taking into account dynamic effects allows better reproducing experimentally observed variation of initiation stress as a function 
of hole size under tensile loading in PMMA holed specimens. The influence of the crack velocity profile under compression loading seems relatively moderate and yields differences smaller than $1 \%$ on initiation crack lengths and loading levels as long as the mean crack velocity remains small, larger differences being obtained for larger mean crack velocities. Finally, the main difficulty in the $\mathrm{CC}$ implementation lies in the knowledge of the crack velocity profile during initiation, which is required as an input in the proposed approach.

\section{References}

[1] A. Griffith, The theory of rupture, in: First International Congress in Applied Mechanics, 1924, pp. 55-63.

[2] G. Irwin, Fracture, Springer Berlin Heidelberg, Berlin, Heidelberg, 1958.

[3] Z. Hashin, Finite thermoelastic fracture criterion with application to laminate cracking analysis, Journal of the Mechanics and Physics of Solids 44 (7) (1996) 1129-1145.

[4] J. Nairn, Exact and variational theorems for fracture mechanics of composites with residual stresses, traction-loaded cracks and imperfect interfaces, International Journal of Fracture 105 (2000) 243-271.

[5] D. Leguillon, Strength or toughness? a criterion for crack onset at a notch, European Journal of Mechanics - A/Solids 21(1) (2002) 61-72.

[6] P. Weißgraeber, D. Leguillon, W. Becker, A review of finite fracture mechanics: crack initiation at singular and non-singular stress raisers, Archive Applied Mechanics 86(1-2) (2016) 375-401.

[7] D. Leguillon, Z. Yosibash, Failure initiation at V-notch tips in quasi-brittle materials, International Journal of Solids and Structures 122-123 (2017) 1-13.

[8] J. Li, D. Leguillon, E. Martin, X. Zhang, Numerical implementation of the coupled criterion for damaged materials, Internation Journal of Solids and Structures 165 (2019) 93-103.

[9] A. Torabi, F. Berto, A. Sapora, Finite fracture mechanics assessment in moderate and large scale yielding regimes, Metals 9 (5) (2019) 602. 
[10] A. Doitrand, A. Sapora, Nonlinear implementation of Finite Fracture Mechanics: A case study on notched Brazilian disk samples, International Journal of Non-Linear Mechanics 119 (2020) 103245.

[11] A. Leite, V. Mantic, F. Paris, Crack onset in stretched open hole pmma plates considering linear and non-linear elastic behaviours, Theoretical and Applied Fracture Mechanics 114 (2021) 102931.

[12] P. Rosendahl, Y. Staudt, A. Schneider, J. Schneider, W. Becker, Nonlinear elastic finite fracture mechanics: modeling mixed-mode crack nucleation in structural glazing silicone sealants, Materials and Design 182 (2019) 108057.

[13] J. Li, D. Leguillon, Finite element implementation of the coupled criterion for numerical simulations of crack initiation and propagation in brittle materials, Theoretical and Applied Fracture Mechanics 93 (2018) 105-115.

[14] A. Doitrand, E. Martin, D. Leguillon, Numerical implementation of the coupled criterion: Matched asymptotic and full finite element approaches, Finite Element in Analysis and Design 168 (2020) 103344.

[15] M. Munoz-Reja, L. Tavara, V. Mantic, P. Cornetti, A numerical implementation of the coupled criterion of finite fracture mechanics for elastic interfaces, Theoretical and Applied Fracture Mechanics 108 (2020) 102607.

[16] E. Martin, T. Vandellos, D. Leguillon, N. Carrère, Initiation of edge debonding: coupled criterion versus cohesive zone model, International Journal of Fracture 199 (2016) 157-168.

[17] R. Dimitri, P. Cornetti, V. Mantic, M. Trullo, L. De Lorenzis, Mode-I debonding of a double cantilever beam: A comparison between cohesive crack modeling and finite fracture mechanics, Internation Journal of Solids and Structures 124 (2017) 57-72.

[18] T. Gentieu, J. Jumel, A. Catapano, J. Broughton, Size effect in particle debonding: comparisons between finite fracture mechanics and cohesive zone model, Journal of Composite Materials 53 (14) (2018) 1941-1954. 
[19] P. Cornetti, M. Munoz-Reja, A. Sapora, A. Carpinteri, Finite fracture mechanics and cohesive crack model: weight functions vs. cohesive laws, International Journal of Solids and Structures 156-157 (2019) 126-136.

[20] A. Doitrand, R. Estevez, D. Leguillon, Comparison between cohesive zone and coupled criterion modeling of crack initiation in rhombus hole specimens under quasi-static compression, Theoretical and Applied Fracture Mechanics 99 (2019) 51-59.

[21] J. Reinoso, A. Arteiro, M. Paggi, P. Camanho, Strength prediction of notched thin ply laminates using finite fracture mechanics and the phase field approach, Composites Science and Technology 150 (2017) 205-216.

[22] M. Strobl, T. Seelig, Phase field modeling of hertzian indentation fracture, Journal of the Mechanics and Physics of Solids 143 (2020) 104026.

[23] G. Molnar, A. Doitrand, R. Estevez, A. Gravouil, Toughness or strength? regularization in phase-field fracture explained by the coupled criterion, Theoretical and Applied Fracture Mechanics 109 (2020) 102736.

[24] A. Doitrand, R. Henry, J. Chevalier, S. Meille, Revisiting the strength of micron-scale ceramic platelets, Journal of the American Ceramic Society 103 (2020) 6991- 7000. doi:10.1111/jace.17148.

[25] A. Doitrand, R. Henry, I. Zacharie-Aubrun, G. J.M., S. Meille, $\mathrm{UO}_{2}$ micron scale specimen fracture : Parameter identification and influence of porosities, Theoretical and Applied Fracture Mechanics 108 (2020) 102665.

[26] A. Doitrand, R. Henry, H. Saad, S. Deville, S. Meille, Determination of interface fracture properties by micro- and macro-scale experiments in nacre-like alumina, Journal of the Mechanics and Physics of Solids 145 (2020) 104143.

[27] P. Gallo, A. Sapora, Brittle failure of nanoscale notched silicon cantilevers: a finite fracture mechanics approach, Applied Science 10(5) (2020) 1640.

[28] Z. Yosibash, B. Mittelman, A 3-D failure initiation criterion from a sharp v-notch edge in elastic brittle structures, European Journal of Mechanics A/Sol. 60 (2016) 70-94. 
[29] I. G. García, B. J. Carter, A. R. Ingraffea, V. Mantič, A numerical study of transverse cracking in cross-ply laminates by 3D finite fracture mechanics, Composites Part B 95 (2016) 475-487.

[30] A. Doitrand, D. Leguillon, 3D application of the coupled criterion to crack initiation prediction in epoxy/aluminum specimens under four point bending, International Journal of Solids and Structures 143 (2018) 175-182.

[31] A. Doitrand, D. Leguillon, Comparison between 2D and 3D applications of the coupled criterion to crack initiation prediction in scarf adhesive joints, International Journal of Adhesion and Adhesives 85 (2018) 69-76.

[32] A. Doitrand, D. Leguillon, Numerical modeling of the nucleation of facets ahead of a primary crack under modeI+III, International Journal of Fracture 123(1) (2018) 37-50.

[33] A. Doitrand, R. Estevez, D. Leguillon, Experimental characterization and numerical modeling of crack initiation in rhombus hole PMMA specimens under compression, European Journal of Mechanics A/Solids 76 (2019) 290-299.

[34] L. Freund, Dynamic Fracture Mechanics, Cambridge university press, 1998.

[35] M. Adda-Bedia, R. Arias, M. Amar, F. Lund, Generalized Griffith criterion for dynamic fracture and the stability of crack motion at high velocities, Physical Review E 60 (2) (1999) 2366.

[36] J. Rice, Some studies of crack dynamics. In: Physical Aspects of Fracture, Springer, 2001.

[37] A. Karma, A. Lobkovsky, Unsteady crack motion and branching in a phase-field model of brittle fracture, Physical Review Letter 92 (24) (2004) 245510.

[38] M. Borden, C. Verhoosel, M. Scott, T. Hughes, C. Landis, A phase-field description of dynamic brittle fracture, Computational Methods in Applied Mechanics and Engineering 217-220 (2012) $77-95$.

[39] G. Molnár, A. Gravouil, R. Seghir, J. Réthoré, An open-source abaqus implementation of the phase-field method to study the effect of plasticity on the instantaneous fracture toughness in dynamic crack propagation, Computer Methods in Applied Mechanics and Engineering 365 (2020) 113004 . 
[40] V. Acary, Y. Monerie, Nonsmooth fracture dynamics using a cohesive zone approach, Tech. rep., Research Report RR-6032, INRIA (2006).

[41] T. Lachuetza, T. Seelig, Remarks on dynamic cohesive fracture under static pre-stress - with a comparison to finite fracture mechanics, Engineering Fracture Mechanics (2021).

[42] K. Ravi-Chandar, W. Knauss, An experimental investigation into dynamic fracture: III. on steady-state crack propagation and crack branching, International Journal of Fracture 26 (1984) 141-154.

[43] E. Sharon, J. Fineberg, Microbranching instability and the dynamic fracture of brittle materials, Phys. Rev. B 54 (1996) 7128-7139. doi:10.1103/PhysRevB.54.7128.

[44] E. Sharon, J. Fineberg, Universal features of the microbranching instability in dynamic fracture, Philosophical Magazine B 78 (2) (1998) 243-251. doi:10.1080/13642819808202947.

[45] A. Livne, G. Cohen, J. Fineberg, Universality and hysteretic dynamics in rapid fracture, Phys. Rev. Lett. 94 (2005) 224301.

[46] K. Broberg, The propagation of a brittle crack, Arkiv Fysik, 1960.

[47] H. Hilber, T. Hughes, R. Taylor, Improved numerical dissipation for time integration algorithms in structural dynamics, Earthquake Engineering and Structural Dynamics 5 (1977) $283-292$.

[48] B. Croquelois, J. Kopp, J. Girardot, P. Tchoreloff, V. Mazel, Dynamic fracture analysis in brazilian test: application to pharmaceutical tablets, International Journal of Fracture 229 (2021) 113-124.

[49] J. Kopp, J. Lin, J. Schmittbuhl, C. Fond, Longitudinal dynamic fracture of polymer pipes, European Journal of Environmental and Civil Engineering 18 (10) (2014) 1097-1105.

[50] K. Kishimoto, S. Aoki, M. Sakata, On the path independent integral-J, Engineering Fracture Mechanics 13 (4) (1980) 841-850.

[51] K. Kishimoto, S. Aoki, M. Sakata, Dynamic stress intensity factors using J-integral and finite element method, Engineering Fracture Mechanics 13 (2) (1980) 387-394. 
[52] A. Parvizi, K. Garrett, J. Bailey, Constrained cracking in glass fibre-reinforced epoxy cross-ply laminates, Journal of Material Science 13 (1978) 195-201.

[53] I. G. García, V. Mantič, A. Blázquez, The effect of residual thermal stresses on transverse cracking in cross-ply laminates: an application of the coupled criterion of the finite fracture mechanics, International Journal of Fracture 211 (2018) 61-74.

[54] P. Soden, M. Hinton, A. Kaddour, A comparison of the predictive capabilities of current failure theories for composite laminates, Composites Science and Technology 58 (1998) 1225-1254.

[55] K. Marlett, Hexcel 8852 as4 unidirectional prepreg at 190 gsm \& 35\% rc qualification material property data report, Tech. rep., National Institute for Aviation Research. Wichita State University, Wichita, Kansas Technical Report CAM-RP-2010-002 Rev A. (2010).

[56] J. Renart, N. Blanco, E. Pajares, J. Costa, S. Lazcano, G. Santacruz, Side clamped beam (scb) hinge system for delamination tests in beam-type composite specimens, Composites Science and Technology 71 (2011) 1023-1029.

[57] J. Li, X. Zhang, A criterion study for non-singular stress concentrations in brittle or quasibrittle materials, Engineering Fracture Mechanics 73 (2006) 505-523.

[58] D. Leguillon, D. Quesada, C. Putot, E. Martin, Size effects for crack initiation at blunt notches or cavities, Engineering Fracture Mechanics 74 (2007) 2420-2436.

[59] E. Martin, D. Leguillon, O. Seveček, R. Bermejo, Understanding the tensile strength of ceramics in the presence of small critical flaws, Engineering Fracture Mechanics 201 (2018) $167-175$.

[60] F. Zhou, J. Molinari, T. Shioya, A rate-dependent cohesive model for simulating dynamic crack propagation in brittle materials, Engineering Fracture Mechanics 72 (2005) 1318-1410.

[61] J. Fineberg, The dynamics of Rapidly moving tensile cracks in brittle amorphous material, Dynamic Fracture Mechanics, 2006.

[62] E. Bura, A. Seweryn, Mode I fracture in pmma specimens with notches - experimental and numerical studies, Theoretical and Applied Fracture Mechanics 97 (2018) 140-155. 
[63] I. Smirnov, N. Kazarinov, Y. Petrov, Experimental observation and numerical modelling of unstable behaviour of a fast crack velocity, Theoretical and Applied Fracture Mechanics 101 (2019) 53-58. 